Article

\title{
Phenotypic Examination of Camelina sativa (L.) Crantz Accessions from the USDA-ARS National Genetics Resource Program
}

\author{
Sara K. Hotton ${ }^{1}$, Meridith Kammerzell ${ }^{2}$, Ron Chan ${ }^{3}$, Bryan T. Hernandez ${ }^{4}$, Hugh A. Young ${ }^{5}$, \\ Christian Tobias ${ }^{3}$, Thomas McKeon ${ }^{3}$, Jenny Brichta ${ }^{3}$, Nathan J. Thomson ${ }^{6}$ and \\ James G. Thomson ${ }^{3, *}$ \\ 1 Bayer Crop Science, West Sacramento, CA 95605, USA; sara.hotton@bayer.com \\ 2 Cargill, Inc., Fort Collins, CO 80525, USA; Meridith_Kammerzell@cargill.com \\ 3 Crop Improvement and Genetics, USDA-ARS-WRRC, Albany, CA 94710, USA; ronald.chan@usda.gov (R.C.); \\ christian.tobias@usda.gov (C.T.); Thomas.McKeon@USDA.GOV (T.M.); jenny.brichta@usda.gov (J.B.) \\ 4 Department of Plant Sciences, University of California, Davis, CA 95616, USA; Rhernandez@sfwater.org \\ 5 Novozyme, Franklinton, NC 27525, USA; HYO@novozymes.com \\ 6 Blinn College, Bryan, TX 77833, USA; Nathan.Thomson31@buc.blinn.edu \\ * Correspondence: James.Thomson@ars.usda.gov
}

Received: 23 March 2020; Accepted: 11 May 2020; Published: 19 May 2020

\begin{abstract}
Camelina sativa (L.) Crntz. is a hardy self-pollinated oilseed plant that belongs to the Brassicaceae family; widely grown throughout the northern hemisphere until the 1940s for production of vegetable oil but was later displaced by higher-yielding rapeseed and sunflower crops. However, interest in camelina as an alternative oil source has been renewed due to its high oil content that is rich in polyunsaturated fatty acids, antioxidants as well as its ability to grow on marginal lands with minimal requirements. For this reason, our group decided to screen the existing (2011) National Genetic Resources Program (NGRP) center collection of camelina for its genetic diversity and provide a phenotypic evaluation of the cultivars available. Properties evaluated include seed and oil traits, developmental and mature morphologies, as well as chromosome content. Selectable marker genes were also evaluated for potential use in biotech manipulation. Data is provided in a raw uncompiled format to allow other researchers to analyze the unbiased information for their own studies. Our evaluation has determined that the NGRP collection has a wide range of genetic potential for both breeding and biotechnological manipulation purposes. Accessions were identified within the NGRP collection that appear to have desirable seed harvest weight $(5.06 \mathrm{~g} / \mathrm{plant})$ and oil content $(44.1 \%)$. Other cultivars were identified as having fatty acid characteristics that may be suitable for meal and/or food use, such as low $(<2 \%)$ erucic acid content, which is often considered for healthy consumption and ranged from a high of $4.79 \%$ to a low of $1.83 \%$. Descriptive statistics are provided for a breadth of traits from 41 accessions, as well as raw data, and key seed traits are further explored. Data presented is available for public use.
\end{abstract}

Keywords: Camelina sativa; oil seed crop; National Genetic Resources Program (NGRP); agronomic traits; biotechnology

\section{Introduction}

Renewable energy sources including esterified vegetable oil (i.e., biodiesel) have been proposed as a possible option to reduce greenhouse gas (GHG) emissions in the transportation sector. Current widely used oilseed for producing biofuel include rapeseed, sunflower (Europe), soybean (USA), and palm oils (tropical regions). In comparison to these other oilseed plants, Camelina sativa (L.) Crntz. (camelina) 
has a very short life cycle (52 to 72 days) and is economical to grow on marginal lands due to minimal nutritional input requirements. It further has the capacity for both summer and winter crop production. The plant is native to northern Europe and Southeast Asia and is a member of the mustard family (Brassicaceae). This ancient crop has previously been used for cooking, cosmetics, and fuel oil [1]. Archaeological studies date its cultivation back to the Bronze Age [2,3]. It was utilized throughout the northern hemisphere, until the 20th century when it was replaced by the high yielding crops, canola, rape, and soy [4,5]. Despite its potential, there is a limitation to more widespread use due to the lack of agronomic knowledge, as well as limited information about the genetic diversity in the available germplasm.

Currently, the crops rapeseed, sunflower, soybean, and palm are primarily used for food and their use in generating oil for biodiesel competes directly with their value as food. Camelina appears an ideal alternative crop for biodiesel as it would not compete with current food applications, contains high oil content that is rich in polyunsaturated fatty acids and tocopherols, which confers stability against oxidation. Due to its oil composition, camelina can be a valuable renewable resource for the production of biodiesel, hydraulic oil, lubricants, and jet fuel [6-9].

Among non-food plants, camelina is well suited for temperate climates with poor soils, thus can be grown on marginal lands. Its beneficial agronomic and economic attributes include good yield under drought conditions, low fertilizer requirement, pesticide demand, compatibility with existing agricultural equipment and short growth time [6-9]. Camelina also displays allelopathic characteristics, discouraging the growth of weeds [10]. With its allelopathic nature, early germination characteristic, and its ability to be sown under freezing conditions, camelina makes an excellent cover-crop. These useful agronomic traits and the fact that camelina has been shown to surpass yields of oilseed crops such as flax under drought-like conditions make it an appealing crop for production in the inland Pacific Northwest and North American semi-arid prairies.

Additionally, the by-product from oil collection provides a meal that is rich in protein and vitamin E, which in turn has prompted studies for its use as an aquaculture and animal feed supplement [11-19]. The meal has been shown to be composed of $45 \%$ protein, $13 \%$ fiber, $5 \%$ vitamins and minerals, as well as $10 \%$ of oil residue [20]. The protein composition includes the amino acids glutamine, asparagine, arginine, leucine, glycine, valine, serine, lysine, and proline in a ratio comparable to rapeseed and soy. However, studies have shown that use of camelina meal in many cases alters the tissue fatty acid composition. It further contains glucosinolates and phytates and therefore must be consumed at low levels. As such, the meal collected from crushed seeds is used as a limited supplement for animal feed [21,22] with poultry and broiler chicken feed rations at a maximum of $10 \%$, beef cattle rations up to $10 \%$, swine feed rations are set at $2 \%$, while Canadian aquaculture is allowed a $3 \%$ ration [23]. However, breeding and or genetic modification offers avenues to improve both the oil and meal composition to meet growing needs.

While the majority to nutritional surveys have been conducted for animal feed there are studies suggesting that camelina oil added to a persons' diet could improve their serum lipid profile [24,25] offering an avenue for its eventual use in the human food stream. However, certain considerations such as low $(<2 \%)$ erucic acid levels $[6,26]$ would need to be addressed by screening, breeding, or biotechnology before widespread utilization occurred. Alternatively, as camelina oil is a rich source of linolenic acid and omega tocopherols, it has potential use in both nutraceuticals and cosmetics [27].

Camelina offers an array of benefits to both the producer and the consumer, therefore exploring untapped genetic reserves appears to be the next logical step. To date, a limited number of camelina lines have been used in field trials, or for biotechnological improvement. Even though there are a large number of cultivars in existence, with tremendous genetic potential, these cultivars have been poorly characterized, and many quality characteristics have not been evaluated at all [28]. Examples of this can be seen in the variation in camelina seed oil content that has been reported to range between 320 and $460 \mathrm{~g} \mathrm{~kg}^{-1}$, and linolenic acid concentrations ranging from 28 to $43 \%$. Results with large seeded genotypes have shown there exists an inverse correlation between 1000 seed weight and oil content 
and therefore these cultivars are considered inferior to small seeded cultivars as an oil source $[7,29]$. Variability also exists for yield with reported ranges from $0.336 \mathrm{tha}^{-1}$ to $2.25 \mathrm{tha}^{-1}$ depending on genotype and location grown $[7,30,31]$. Although, these results could be and are most likely due to environmental factors as well as genetic, more studies will be needed using true breeding populations of camelina with a large set of known QTLs to determine.

Currently, camelina stands on the brink of multiple industrial applications and potential animal feed use. Above and beyond its apparent native potential is the fact that this species can be genetically manipulated with relative ease $[8,23,32-38]$. Published results of a selection of germplasm treated to a mutagenesis technique has led to camelina cultivars with higher oil content and improved fatty acid composition, such as the cultivar Blaine Creek that is enriched in $\omega-3$ fatty acids, and Suneson, which has shown 2-3\% higher oil content with enhanced $\alpha$-linolenic acid $[39,40]$.

Another option is the use of direct genomic modification utilizing biotechnological techniques. Being a close relative of Arabidopsis, there are 30 years of molecular biology techniques for genetic modification available for use [41-43]. To date, genome modification has been used to enhance the metabolic pathway for fatty acid production in order to enhance production levels [32,33,44-47]. Transcriptome analysis and comparative genomics have provided a wealth of information for both breeders and biotechnological applications [48-54]. Sophisticated molecular techniques for genome modification such as RNAi [21] and CRISPR [55] have been utilized for reducing or abolishing gene expression to shift the fatty acid metabolic process. While techniques such as recombinase mediated cassette exchange (RMCE) [56] or gene assembly in agrobacterium by nucleic acid transfer using recombinase technology (GAANTRY) [57] could be employed for addition of large genetic pathways for novel metabolic engineering projects. These modifications could be used to tailor camelina oil or meal for specific uses [58-63]. An end goal would be, for these plant-based oils, to become an option to replace non-sustainable petroleum-based products as fuel, lubricants and specialty chemicals. For a review of biotechnological improvements to Camelina, see Bansal et al. [64].

However, even with the advances seen in molecular modification certain basic biotechnological tools are still lacking, such as selectable markers genes. To date the herbicide bar selection system is the only robust selection method available. Others marker genes such as hygromycin (hptII) [43] and acetolactate synthase (ALS) [42,63-65] have been published, but do not appear as robust as bar, and therefore may have limited use as a biotechnological tool.

Genetic and other background information about the origin of particular camelina genotypes is essential for initiating focused breeding programs [66] but is not available or cross referenced for most of the available National Genetic Resources Program (NGRP) accessions. The aim of the present study has been to provide an overview of the phenotypic diversity present within the NGRP camelina germplasm collection, as of 2011. We attempt to capture and present agronomically important traits and collate this information with accessible and searchable NGRP camelina accessions. Data is presented on 41 cultivars that include traits such as the range of germination rates, time to bolt, heritable seed traits-such as seed size, 1000-seed weight, oil content, fatty acid-as well as chromosome counts. We further provide information on the use of novel selection marker genes for potential utilization in biotechnological applications. Data is presented in an uncompressed format in both tables for manuscript discussion and as raw data in XLS spreadsheet (Supplemental Tables S1-S4) format.

\section{Results}

Identifying camelina plant and seed quality traits for important agronomic characteristics for marketing and processing is needed to develop this crop which is in direct competition with other oilseeds currently in production. Therefore, we measured a number of traits that include 1000-seed weight, oil content, and performed compositional analysis. Agronomic characteristics were also monitored for the different genotypes under controlled conditions in order to minimize environmental factors and evaluate genetic influences and to catalogue phenotypic responses. Our lab initially obtained 38 camelina accessions from the NGRP for evaluation but four of the lines (PI 597833, 
PI 650142, PI 650157, PI 650158) were discovered to have mixed seed from two distinct cultivars. These lines were therefore given designations of ' $A$ ' and ' $\mathrm{B}$ ' types of the originating name. The NGRP collection cultivar PI 304268 appeared to require vernalization conditions that were not met in this study, as such, the plant remained in the rosette stage, failing to flower and provide seed. Therefore, this line was not included in this study; giving a total of 41 accessions reviewed.

\subsection{Plant Description}

Camelina is a self-pollinated plant with small, 4-lobed flowers of pale yellow. The tear shaped fruits, or siliques, appear similar to flax bolls and contain approximately 15-20 small seeds with a high oil content, making it desirable for potential commercialization. Throughout the study, phenotypic descriptions and growth stages of camelina sativa presented will be subtitled according to the two-digit BBCH scale [67] in the text.

\subsection{Early Development}

Principal growth stage, seed germination, and early development. 01: Initiation of seed imbibition-It was observed that all viable seed for every cultivar formed a mucosal/gelatinous coat within the first $5 \mathrm{~min}$ of being imbibed. 03: Radicle emergence from seed-All cultivars showed radical emergence in 1 to 2 days with a median rate of 1 day (Table 1). 04: Emergence of hypocotyl with cotyledons from the seed-Shoot emergence was seen from 1.8 to 3 days after sowing on damp soil and with a median rate of 2 days (Table 1). At day 4, cultivars were measured for root length, hypocotyl length, and percent cotyledon unfolding. Roots were found to range from $5.4 \mathrm{~mm}$ to $40.4 \mathrm{~mm}$ with a median value of $25.9 \mathrm{~mm}$ (Table 1). Hypocotyl length was determined to have values from $2.2 \mathrm{~mm}$ to $10.8 \mathrm{~mm}$ with a median value of $7.8 \mathrm{~mm}$ (Table 1). 10: Cotyledons (node 0 ) unfolded-The rate of cotyledon unfolding was measured at day 4 and was observed to range from 0 to $100 \%$ with a median value of $72 \%$. Vernalization was required for 9 of the 41 cultivars and these are indicated by asterisks at the end of their accession number (Table 1).

Table 1. Early emergence.

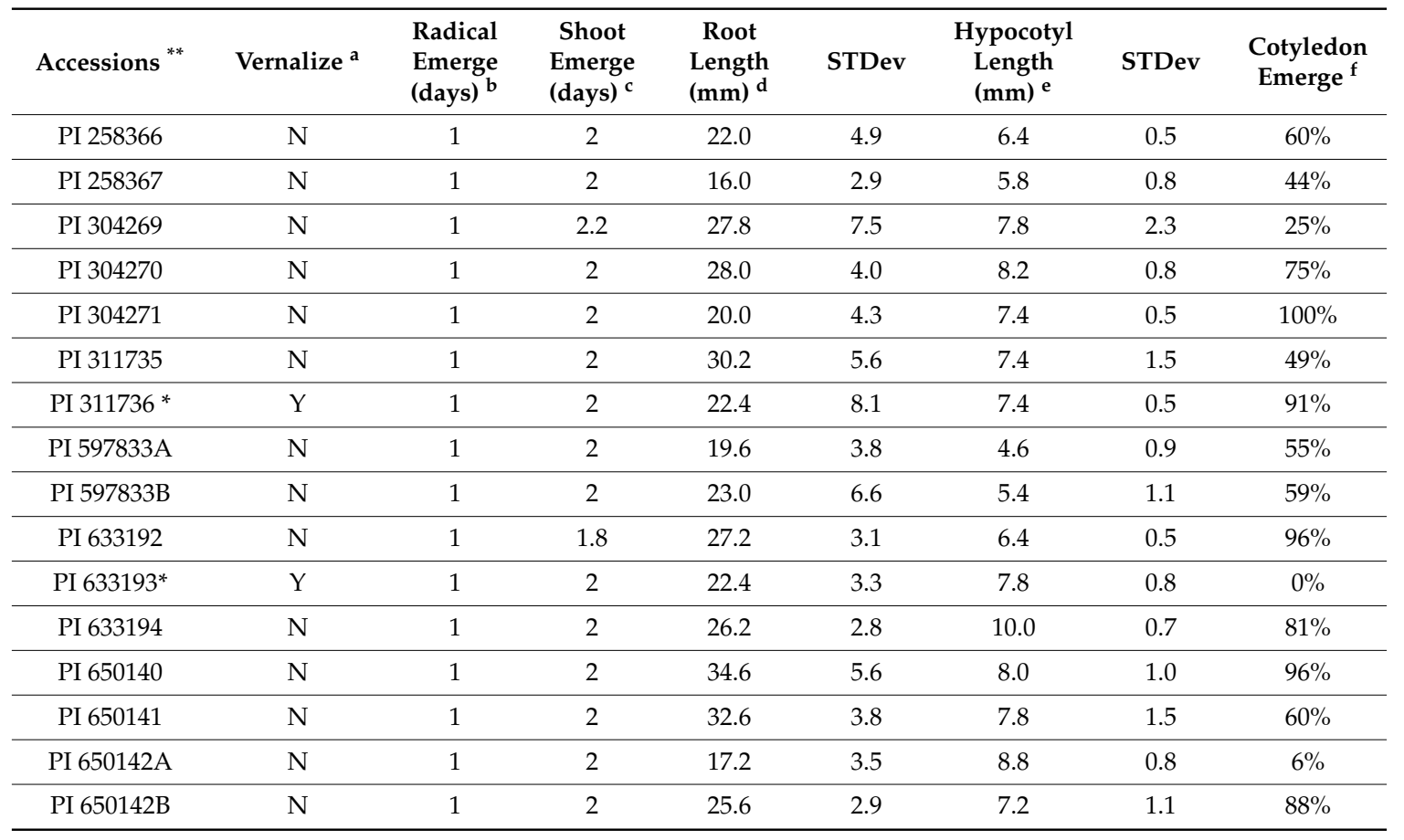


Table 1. Cont.

\begin{tabular}{|c|c|c|c|c|c|c|c|c|}
\hline Accessions ${ }^{* *}$ & Vernalize $^{a}$ & $\begin{array}{l}\text { Radical } \\
\text { Emerge } \\
\text { (days) }^{b}\end{array}$ & $\begin{array}{l}\text { Shoot } \\
\text { Emerge } \\
\text { (days) }^{c}\end{array}$ & $\begin{array}{l}\text { Root } \\
\text { Length } \\
(\mathrm{mm})^{d}\end{array}$ & STDev & $\begin{array}{l}\text { Hypocotyl } \\
\text { Length } \\
(\mathrm{mm})^{\mathrm{e}}\end{array}$ & STDev & $\begin{array}{l}\text { Cotyledon } \\
\text { Emerge }\end{array}$ \\
\hline PI 650143 * & $\mathrm{Y}$ & 1 & 2 & 5.4 & 1.5 & 2.8 & 0.8 & $3 \%$ \\
\hline PI 650144 & $\mathrm{~N}$ & 1 & 2.2 & 24.6 & 11.1 & 7.4 & 1.5 & $70 \%$ \\
\hline PI 650145 & $\mathrm{~N}$ & 1 & 2 & 30.4 & 9.6 & 8.2 & 0.8 & $29 \%$ \\
\hline PI 650146 & $\mathrm{~N}$ & 1 & 2 & 23.0 & 6.7 & 8.4 & 1.1 & $61 \%$ \\
\hline PI 650147 & $\mathrm{~N}$ & 1 & 2 & 20.2 & 4.8 & 9.0 & 0.7 & $24 \%$ \\
\hline PI 650148 & $\mathrm{~N}$ & 1 & 2 & 23.0 & 3.3 & 7.6 & 0.5 & $100 \%$ \\
\hline PI 650149 & $\mathrm{~N}$ & 1 & 2 & 26.8 & 6.6 & 8.2 & 0.8 & $77 \%$ \\
\hline PI 650150 & $\mathrm{~N}$ & 1 & 2 & 38.2 & 4.3 & 8.6 & 0.5 & $85 \%$ \\
\hline PI 650151 & $\mathrm{~N}$ & 1 & 2 & 33.4 & 3.5 & 9.0 & 1.0 & $75 \%$ \\
\hline PI 650152 * & $\mathrm{Y}$ & 1 & 2 & 8.8 & 1.3 & 2.2 & 0.4 & $22 \%$ \\
\hline PI 650153 & $\mathrm{~N}$ & 1 & 2 & 38.0 & 7.2 & 8.8 & 1.3 & $0 \%$ \\
\hline PI 650154 & $\mathrm{~N}$ & 1 & 2 & 26.4 & 2.8 & 8.0 & 0.7 & $96 \%$ \\
\hline PI 650155 * & $\mathrm{Y}$ & 1 & 2 & 17.6 & 3.5 & 7.0 & 1.0 & $98 \%$ \\
\hline PI 650156 & $\mathrm{~N}$ & 1 & 2 & 31.2 & 6.3 & 9.4 & 0.5 & $100 \%$ \\
\hline PI 650157A * & $\mathrm{Y}$ & 1 & 2 & 22.6 & 2.7 & 5.4 & 0.5 & $69 \%$ \\
\hline PI 650157B & $\mathrm{N}$ & 1 & 2 & 24.2 & 1.9 & 5.4 & 1.1 & $14 \%$ \\
\hline PI 650158A * & $\mathrm{Y}$ & 1 & 2 & 40.4 & 5.1 & 7.4 & 0.5 & $88 \%$ \\
\hline PI 650158B & $\mathrm{N}$ & 1 & 2 & 26.2 & 6.3 & 8.2 & 0.8 & $90 \%$ \\
\hline PI 650159 & $\mathrm{~N}$ & 1 & 2 & 37.4 & 15.1 & 7.8 & 1.3 & $80 \%$ \\
\hline PI 650163 & $\mathrm{~N}$ & 1.2 & 1.8 & 22.0 & 2.7 & 7.2 & 1.3 & $24 \%$ \\
\hline PI 650164 & $\mathrm{~N}$ & 1 & 2 & 30.8 & 7.0 & 8.6 & 1.1 & $100 \%$ \\
\hline PI 650165 & $\mathrm{~N}$ & 1 & 2 & 36.8 & 13.8 & 8.8 & 1.8 & $94 \%$ \\
\hline PI 650166 & $\mathrm{~N}$ & 1 & 2 & 28.4 & 5.9 & 8.0 & 1.2 & $7 \%$ \\
\hline PI 650167 * & $\mathrm{Y}$ & 2 & 3 & 13.8 & 1.5 & 3.8 & 0.8 & $66 \%$ \\
\hline PI 650168 * & Y & 1 & 2 & 20.6 & 3.6 & 5.6 & 0.5 & $89 \%$ \\
\hline Suneson & $\mathrm{N}$ & 1 & 2 & 29.8 & 10.8 & 10.8 & 0.8 & $75 \%$ \\
\hline
\end{tabular}

\subsection{Physical Attributes}

Principal growth stage 1: leaf development. Leaf morphology was observed in three basic shapes with lanceolate $(75.6 \%)$ being the most predominant, followed by subulate $(17.1 \%)$ and linear $(7.3 \%)$ (Figure 1, Table 2). Leaf margin morphology (shape of the edge) was determined to also have three basic characteristics with spiny $(41.5 \%)$ most often observed. Serrate $(29.3 \%)$ was the second most common followed by smooth (26.8\%). Examples of shapes are seen in Figure 1 and each accession's combination of leaf shape, edge shape, and spine number (points) characteristics are listed in Table 2. Like most of the Brassicaceae, camelina develops lateral branches. The development of lateral branches is variable and depends on the genotype. Plant density and environmental conditions that may also affect the number of branches [67]. Here we present findings of lateral branch development under low plant density and favorable growth (greenhouse) conditions. 
Shape

Lanceolate $75.6 \%$

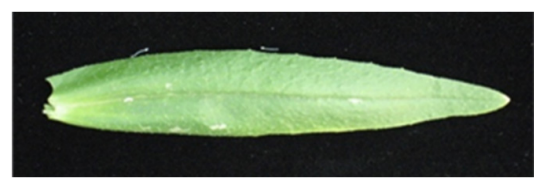

Subulate $17.1 \%$

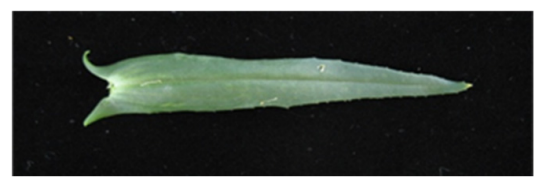

Linear 7.3\%

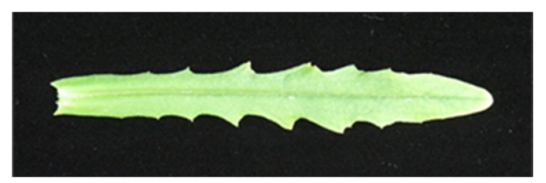

\section{Margin}

Spiny $41.5 \%$

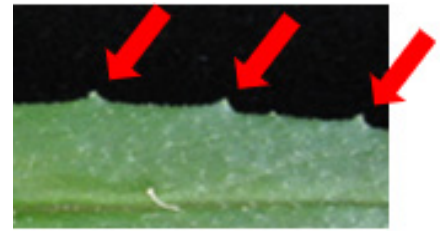

Serrate $29.3 \%$

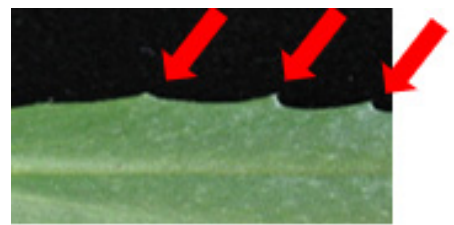

Smooth $26.8 \%$

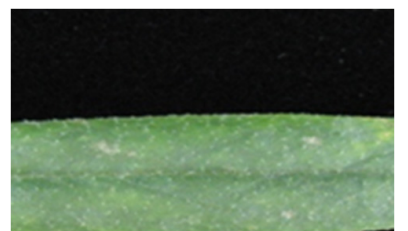

Figure 1. Leaf shape and margin. Shape and margin of the leaf were determined after plants had bolted and reached mature height. Lanceolate (narrow oval shape tapering to a point at each end) was seen in $75.6 \%$ of population. Subulate (slender and tapering to a point) was observed $17.1 \%$ and Linear (long and very narrow like a blade of grass) seen 7.3\%. The Spiny (stiff, sharp points such as thistles) margin was most prevalent in the population at $41.5 \%$. Serrated (saw-toothed; with asymmetrical teeth pointing forward) was seen $29.3 \%$ and Smooth (even margin without points) was $26.8 \%$. Points are small needle like projections seen at the leaf edge seen in the spiny and serrated examples with red arrows above.

Table 2. Branch and leaf morphology.

\begin{tabular}{|c|c|c|c|c|c|}
\hline Accession $^{* *}$ & Leaf Shape & Leaf Margin a $^{a}$ & Leaf Points $b$ & Total Points & Branch Pattern $^{c}$ \\
\hline PI 258366 & subulate & serrate & $4+4$ & 8 & $\mathrm{~W}$ \\
\hline PI 258367 & lanceolate & smooth & NA & NA & $\mathrm{W}$ \\
\hline PI 304269 & lanceolate & smooth & NA & NA & $\mathrm{W}$ \\
\hline PI 304270 & lanceolate & spiny & $7+7$ & 14 & $\mathrm{~W}$ \\
\hline PI 304271 & lanceolate & spiny & $5+5$ & 10 & $\mathrm{~W}$ \\
\hline PI 311735 & lanceolate & spiny & $6+5$ & 11 & $\mathrm{~W}$ \\
\hline PI $311736^{*}$ & lanceolate & mildly serrate & $6+6$ & 12 & $\mathrm{Y}$ \\
\hline PI 597833A & linear & serrated & $9+9$ & 18 & $\mathrm{~W}$ \\
\hline PI 597833B & lanceolate & spiny & $6+5$ & 11 & $\mathrm{~W}$ \\
\hline PI 633192 & lanceolate & spiny & $6+6$ & 12 & $\mathrm{~W}$ \\
\hline PI $633193 *$ & lanceolate & smooth & NA & NA & $x$ \\
\hline PI 633194 & lanceolate & spiny & $7+8$ & 15 & $\mathrm{~W}$ \\
\hline PI 650140 & lanceolate & spiny & $5+5$ & 10 & $x$ \\
\hline PI 650141 & lanceolate & spiny & $6+5$ & 11 & $\mathrm{~W}$ \\
\hline PI 650142A & linear & serrate & $7+6$ & 13 & $\mathrm{~W}$ \\
\hline PI 650142B & lanceolate & spiny & $6+6$ & 12 & $\mathrm{~W}$ \\
\hline
\end{tabular}


Table 2. Cont.

\begin{tabular}{|c|c|c|c|c|c|}
\hline Accession $^{* *}$ & Leaf Shape & Leaf Margin $^{a}$ & Leaf Points $b$ & Total Points & Branch Pattern ${ }^{c}$ \\
\hline PI $650143 *$ & subulate & mildly serrate & $6+6$ & 12 & $x$ \\
\hline PI 650144 & lanceolate & mildly serrate & $8+8$ & 16 & $\mathrm{~W}$ \\
\hline PI 650145 & lanceolate & smooth & NA & NA & $\mathrm{W}$ \\
\hline PI 650146 & lanceolate & smooth & NA & NA & $\mathrm{W}$ \\
\hline PI 650147 & lanceolate & spiny & $6+7$ & 13 & $\mathrm{~W}$ \\
\hline PI 650148 & lanceolate & smooth & NA & NA & $\mathrm{W}$ \\
\hline PI 650149 & lanceolate & spiny & $8+7$ & 15 & $\mathrm{~W}$ \\
\hline PI 650150 & lanceolate & smooth & NA & NA & $\mathrm{W}$ \\
\hline PI 650151 & lanceolate & smooth & NA & NA & $\mathrm{W}$ \\
\hline PI $650152 *$ & lanceolate & serrate & $7+7$ & 14 & $x$ \\
\hline PI 650153 & lanceolate & spiny & $5+5$ & 10 & $\mathrm{~W}$ \\
\hline PI 650154 & lanceolate & spiny & $5+5$ & 10 & $\mathrm{~W}$ \\
\hline PI $650155^{*}$ & subulate & mildly serrate & $7+7$ & 14 & $\mathrm{~W}$ \\
\hline PI 650156 & lanceolate & serrate & $9+9$ & 18 & $\mathrm{~W}$ \\
\hline PI 650157A * & subulate & mildly serrate & $8+8$ & 16 & $x$ \\
\hline PI 650157B & subulate & smooth & NA & NA & $\mathrm{Z}$ \\
\hline PI 650158A* & lanceolate & spiny & $7+7$ & 14 & $\mathrm{~W}$ \\
\hline PI 650158B & lanceolate & smooth & NA & NA & $\mathrm{W}$ \\
\hline PI 650159 & lanceolate & mildly serrate & $6+5$ & 11 & $x$ \\
\hline PI 650163 & subulate & spiny & $13+21$ & 34 & $\mathrm{~W}$ \\
\hline PI 650164 & lanceolate & serrate & $6+7$ & 13 & $\mathrm{Y}$ \\
\hline PI 650165 & subulate & spiny & $3+4$ & 7 & $\mathrm{~W}$ \\
\hline PI 650166 & lanceolate & spiny & $9+5$ & 14 & $\mathrm{~W}$ \\
\hline PI $650167^{*}$ & lanceolate & smooth & NA & NA & $\mathrm{W}$ \\
\hline PI $650168 *$ & lanceolate & mildly serrate & $6+6$ & 12 & $\mathrm{Z}$ \\
\hline Suneson & lanceolate & spiny & $8+6$ & 14 & $\mathrm{~W}$ \\
\hline
\end{tabular}

* Indicates accessions that required vernalization in order to flower. Also described as 'winter' accessions. ** A sample size $(n)$ of 3-12 was used per cultivar. See Supplemental Table S2 for specific values. A and B designation were given to accession obtained from the NGRP center that contained more than two unique phenotypes. ${ }^{\text {a }}$ Spiny margin, defined as having a series of sharp stiff points; serrated margin, defined as having a series of wave like forward pointed teeth around the entire leaf edge; smooth margin, defined as no projections around the outside of the leaf. ${ }^{b}$ Points are defined as short needle like projections or spines from the edge of the leaf. ' $4+4$ ' defined as points on 'right' side of leaf + points on 'left' side of leaf. ${ }^{\mathrm{c}} \mathrm{W}$-Top heavy branching often seen with secondary branching; $X$-Branched length of main stem with few secondary branches; $Y$-Tiller where all branches originate from the base and contain few secondary branching; $Z$-Chaos where branches are seen originating everywhere and a dominant stem was not observed.

Lateral branch development appears to have four unique patterns. Top heavy branching (W-75.6\%) often seen with secondary branching is by far the most common, followed by branched length of main stem $(X-14.6 \%)$ with few secondary branches observed, Tiller $(Y-4.9 \%)$ where all branches originate from the base and contain few secondary branching and finally Chaos $(\mathrm{Z}-4.9 \%)$ where branches are seen originating everywhere without a dominant stem observed (Figure 2, Table 2). Interestingly, four cultivars PI 311736, PI 650143, PI 650152, and PI 650167 appeared to keep the rosette throughout their lifecycle, under greenhouse conditions. All appear to be winter cultivars. 


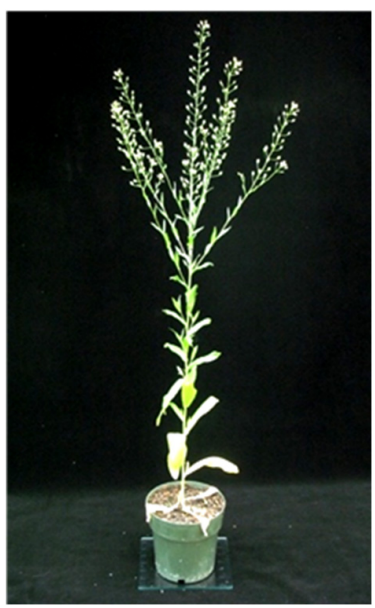

W type

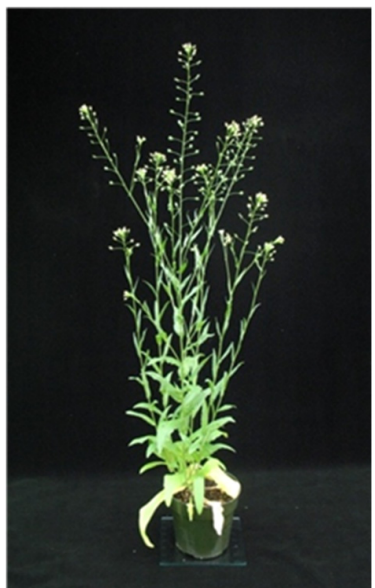

Y type

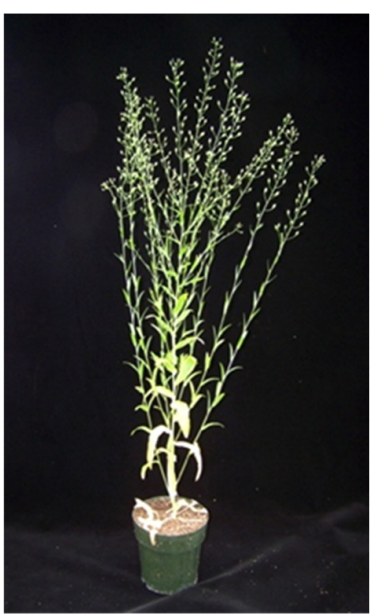

$14.6 \%$

$(6 / 41)$

X type

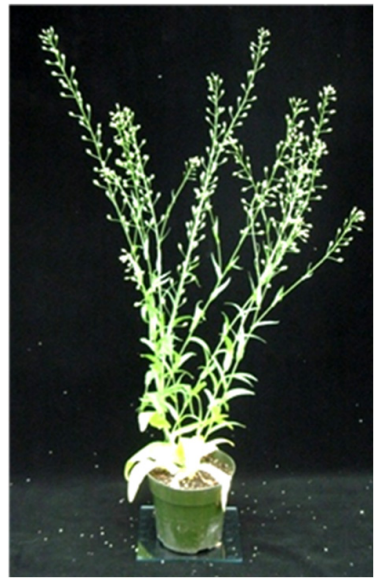

$4.9 \%$

$(2 / 41)$

Figure 2. Branch types. W type-branched only at top and seen in $75.6 \%$ of population. Cultivar PI 597833B shown. X type-branched down length of main stem. Observed in $14.6 \%$ of population; PI 25366 depicted. Y type-tiller like; most all branches originating from the base. Seen in $4.9 \%$ of the population; PI 311736 pictured. Z type-chaos; no main stem observed and secondary branches emerging everywhere. Observed in 4.9\% of cultivars; PI 650168 shown as an example.

The cultivars in this study showed a range of bolting time from 18 to 35 days with an average of 23 days (Table 3). Principal growth stage 6: flowering (main shoot); 65: Full flowering: 50\% of flowers open-The primary inflorescence is a composite flower composed of the number of florets and defined as 'open' when greater than $50 \%$ of the florets had unfolded. This occurred in a minimum of 29 days and a maximum of 43 days with the median being 33 days (Table 3). The number of leaves present on the primary bolting stem at time of flowering ranged from 18 to 41 (Table 3). The mean value height and width (Table 3) seen at the time of initial flowering were $45 \mathrm{~cm}$ and $22 \mathrm{~cm}$, respectively. The primary inflorescence contains 9 florets at a minimum and 18 at a maximum with a median value of 12 , while the secondary inflorescence contains an average of 4 florets (Table 3). 
Table 3. Flowering development.

\begin{tabular}{|c|c|c|c|c|c|c|c|c|c|c|}
\hline Accession ${ }^{* *}$ & $\begin{array}{l}\text { Bolting } \\
\text { (days) }^{a}\end{array}$ & STDev & $\begin{array}{c}\text { Flowering } \\
\text { (days) }^{b}\end{array}$ & STDev & $\begin{array}{l}\text { Height } \\
(\mathrm{cm})^{\mathrm{c}}\end{array}$ & STDev & $\begin{array}{l}\text { Width } \\
\text { (cm) })^{d}\end{array}$ & STDev & $\begin{array}{c}1^{\circ} \text { Floral } \\
\text { Meristem }^{\mathrm{e}}\end{array}$ & STDev \\
\hline PI 258366 & 21.0 & 0.0 & 29.8 & 1.1 & 48.3 & 3.3 & 22.6 & 1.9 & 18.4 & 3.3 \\
\hline PI 258367 & 20.3 & 1.4 & 34.3 & 4.5 & 49.4 & 5.1 & 18.9 & 4.0 & 12.9 & 2.7 \\
\hline PI 304269 & 23.0 & 0.0 & 37.4 & 1.3 & 50.9 & 6.1 & 21.6 & 1.5 & 13.2 & 1.6 \\
\hline PI 304270 & 23.0 & 0.0 & 37.4 & 1.3 & 50.9 & 6.0 & 22.6 & 1.5 & 13.2 & 2.2 \\
\hline PI 304271 & 21.0 & 0.0 & 29.0 & 0.0 & 49.6 & 3.8 & 23.0 & 2.0 & 13.4 & 1.1 \\
\hline PI 311735 & 18.1 & 1.2 & 32.6 & 3.5 & 50.7 & 8.8 & 18.1 & 4.9 & 10.1 & 4.2 \\
\hline PI 311736 * & 24.0 & 0.0 & 30.6 & 4.3 & 30.8 & 14.0 & 25.1 & 0.5 & 11.0 & 2.9 \\
\hline PI 597833A & 21.0 & 0.0 & 32.3 & 4.9 & 38.8 & 7.5 & 20.6 & 2.8 & 10.3 & 1.2 \\
\hline PI 597833B & 20.0 & 2.1 & 33.0 & 3.0 & 46.8 & 8.5 & 18.2 & 3.7 & 9.6 & 2.2 \\
\hline PI 633192 & 23.0 & 4.0 & 31.3 & 4.5 & 36.4 & 4.5 & 24.3 & 2.1 & 13.3 & 2.9 \\
\hline PI 633193 * & 19.2 & 2.7 & 33.2 & 4.9 & 43.5 & 10.3 & 17.4 & 5.9 & 14.4 & 5.7 \\
\hline PI 633194 & 19.9 & 1.6 & 33.9 & 4.2 & 50.0 & 3.5 & 17.8 & 2.6 & 10.1 & 4.2 \\
\hline PI 650140 & 20.6 & 1.1 & 32.4 & 3.2 & 49.5 & 7.1 & 20.9 & 2.3 & 11.9 & 2.1 \\
\hline PI 650141 & 21.0 & 0.0 & 29.0 & 0.0 & 41.0 & 5.1 & 18.8 & 0.8 & 11.0 & 1.6 \\
\hline PI 650142A & 21.8 & 1.0 & 31.3 & 3.1 & 38.5 & 4.1 & 22.8 & 1.5 & 12.0 & 1.9 \\
\hline PI 650142B & 22.0 & 1.3 & 33.3 & 3.7 & 45.6 & 8.8 & 21.6 & 1.6 & 12.4 & 1.7 \\
\hline PI $650143 *$ & 35.0 & 0.0 & 38.0 & 0.0 & 32.5 & 0.7 & 20.0 & 2.8 & 9.0 & 0.0 \\
\hline PI 650144 & 24.3 & 3.2 & 38.0 & 2.8 & 47.4 & 6.9 & 20.8 & 4.8 & 10.8 & 1.4 \\
\hline PI 650145 & 23.0 & 0.0 & 35.6 & 1.3 & 44.2 & 4.4 & 20.8 & 1.1 & 10.6 & 0.5 \\
\hline PI 650146 & 23.0 & 0.0 & 38.6 & 2.5 & 49.0 & 6.0 & 23.0 & 3.7 & 10.2 & 1.9 \\
\hline PI 650147 & 23.0 & 0.0 & 38.6 & 1.3 & 48.6 & 1.5 & 24.4 & 1.7 & 12.4 & 1.1 \\
\hline PI 650148 & 23.0 & 0.0 & 41.2 & 4.1 & 47.0 & 4.6 & 18.8 & 3.4 & 12.2 & 1.9 \\
\hline PI 650149 & 27.0 & 0.0 & 42.8 & 1.6 & 50.3 & 8.2 & 23.0 & 0.7 & 14.8 & 1.6 \\
\hline PI 650150 & 23.0 & 0.0 & 36.2 & 1.6 & 40.2 & 6.2 & 22.6 & 4.0 & 9.2 & 0.8 \\
\hline PI 650151 & 23.0 & 0.0 & 36.8 & 1.6 & 56.5 & 10.8 & 25.6 & 1.5 & 11.0 & 1.0 \\
\hline PI $650152 *$ & 30.0 & 3.1 & 37.4 & 1.3 & 46.8 & 8.9 & 25.0 & 5.9 & 12.6 & 1.8 \\
\hline PI 650153 & 20.0 & 0.0 & 33.6 & 3.3 & 51.6 & 16.1 & 22.8 & 0.8 & 13.2 & 4.1 \\
\hline PI 650154 & 20.0 & 0.0 & 36.0 & 0.0 & 60.6 & 10.9 & 19.8 & 1.5 & 14.2 & 4.5 \\
\hline PI $650155^{*}$ & 21.0 & 0.0 & 29.2 & 1.6 & 39.2 & 7.0 & 26.6 & 2.3 & 10.4 & 1.3 \\
\hline PI 650156 & 20.6 & 0.5 & 35.4 & 0.5 & 54.4 & 8.0 & 18.4 & 4.3 & 10.3 & 2.2 \\
\hline PI 650157A * & 21.6 & 1.3 & 28.6 & 1.3 & 28.6 & 7.1 & 21.6 & 2.7 & 11.0 & 3.1 \\
\hline PI 650157B & 24.0 & 1.9 & 33.5 & 2.8 & 40.7 & 9.7 & 15.8 & 1.6 & 10.0 & 1.5 \\
\hline PI 650158A* & 21.0 & 0.0 & 31.0 & 0.0 & 36.6 & 5.6 & 25.2 & 3.8 & 10.6 & 3.3 \\
\hline PI 650158B & 22.2 & 2.7 & 31.2 & 3.9 & 47.2 & 3.4 & 22.8 & 2.9 & 9.4 & 0.9 \\
\hline PI 650159 & 20.0 & 0.0 & 37.0 & 2.2 & 62.1 & 6.4 & 23.8 & 1.9 & 11.2 & 1.6 \\
\hline PI 650163 & 22.4 & 4.4 & 29.8 & 1.1 & 44.3 & 14.9 & 24.0 & 1.0 & 11.6 & 2.9 \\
\hline PI 650164 & 21.0 & 0.0 & 29.0 & 0.0 & 39.7 & 5.5 & 22.2 & 2.2 & 11.4 & 1.1 \\
\hline PI 650165 & 21.0 & 0.0 & 29.0 & 0.0 & 58.4 & 4.3 & 20.8 & 1.3 & 16.0 & 2.1 \\
\hline PI 650166 & 21.0 & 0.0 & 33.2 & 3.8 & 43.1 & 7.8 & 17.7 & 2.7 & 12.4 & 3.9 \\
\hline PI $650167^{*}$ & 33.8 & 3.4 & 39.0 & 2.0 & 23.0 & 8.8 & 22.3 & 2.6 & 9.8 & 1.5 \\
\hline PI $650168 *$ & 23.0 & 1.7 & 29.0 & 1.7 & 29.7 & 6.5 & 24.3 & 0.6 & 11.7 & 2.1 \\
\hline Suneson & 22.1 & 1.2 & 36.8 & 4.1 & 50.5 & 4.2 & 20.8 & 5.0 & 12.0 & 3.5 \\
\hline
\end{tabular}

${ }^{*}$ Indicates accessions that required vernalization in order to flower. Also described as 'winter' accessions. ${ }^{* *}$ A sample size (n) of 3-12 was used per cultivar. See Supplemental Table S2 for specific values. A and B designation were given to accession obtained from the NGRP center that contained more than two unique phenotypes. ${ }^{a}$ Defined as the rapid development of the first flower stalk or central stem. ${ }^{b}$ Defined as time when greater than $50 \%$ of all floret within the initial composite flower are open. ${ }^{c}$ Maximum height of plant as determined at initial flowering. ${ }^{d}$ Maximum width of plant as determined at initial flowering. ${ }^{e}$ Primary inflorescence is composed of a number of florets (one of the small flowers) making up a composite flower head. 
Maturity of the plant was defined as the time when the plant is in full flower, it has reached its maximum height and seed set has begun. The earliest accessions to reach maturity were PI 650164 and PI 650165 at 42 days and the latest at 63 day was seen by PI 650143. A median maturity value of 50 days was observed (Table 4). 39: Maximum stem length-Accessions reached maximum height at maturity, which was seen to be between $64.9 \mathrm{~cm}$ (PI 650142A) to a maximum of $88.3 \mathrm{~cm}$ (PI 650151) with a median value of $78.3 \mathrm{~cm}$ (Table 4$)$. The plants width at maturity was observed to be between $11.4 \mathrm{~cm}$ (PI 650153) to $61.0 \mathrm{~cm}$ (PI 650168) with a median value of $36.6 \mathrm{~cm}$ (Table 4). Principal growth stage 9: senescence. 97: Plant dead and dry-Plants were dry and seeds ready to harvest at a minimum of 52 days (PI 304271, PI 650164, PI 650165), at a maximum of 72 days (PI 650145, PI 650146) with an mean value of 63 days (Table 4 ).

Table 4. Plant development.

\begin{tabular}{|c|c|c|c|c|c|c|c|c|}
\hline Accession ${ }^{* *}$ & $\begin{array}{l}\text { Maturity } \\
\text { (days) }^{a}\end{array}$ & STDev & $\begin{array}{l}\text { Height } \\
(\mathrm{cm})^{b}\end{array}$ & STDev & $\begin{array}{l}\text { Width } \\
(\mathrm{cm})^{c}\end{array}$ & STDev & $\begin{array}{c}\text { Dry } \\
\text { (days) }^{d}\end{array}$ & STDev \\
\hline PI 258366 & 43.2 & 1.6 & 80.4 & 4.2 & 39.8 & 7.2 & 53.6 & 2.2 \\
\hline PI 258367 & 48.3 & 5.2 & 79.1 & 5.5 & 41.8 & 6.4 & 54.7 & 2.3 \\
\hline PI 304269 & 49.8 & 1.6 & 82.5 & 1.4 & 32.8 & 3.4 & 62.0 & 0.0 \\
\hline PI 304270 & 50.4 & 1.3 & 83.4 & 3.4 & 38.6 & 4.3 & 62.0 & 0.0 \\
\hline PI 304271 & 42.0 & 0.0 & 76.6 & 2.7 & 38.4 & 5.5 & 52.0 & 0.0 \\
\hline PI 311735 & 48.3 & 5.2 & 74.3 & 6.8 & 33.1 & 8.8 & 60.8 & 7.2 \\
\hline PI 311736 * & 52.0 & 0.0 & 71.4 & 6.9 & 49.8 & 13.8 & 66.0 & 0.0 \\
\hline PI 597833A & 50.3 & 4.1 & 67.2 & 5.5 & 44.2 & 4.4 & 63.7 & 5.7 \\
\hline PI 597833B & 48.3 & 5.2 & 80.4 & 6.8 & 39.0 & 4.0 & 60.8 & 7.2 \\
\hline PI 633192 & 44.5 & 5.0 & 72.5 & 3.7 & 31.8 & 5.7 & 53.8 & 3.5 \\
\hline PI $633193 *$ & 52.0 & 0.0 & 73.0 & 9.2 & 57.4 & 21.1 & 66.0 & 0.0 \\
\hline PI 633194 & 48.3 & 5.2 & 71.8 & 7.3 & 35.9 & 8.7 & 64.4 & 10.3 \\
\hline PI 650140 & 47.7 & 5.3 & 77.0 & 6.8 & 48.6 & 15.2 & 60.0 & 7.5 \\
\hline PI 650141 & 42.0 & 0.0 & 74.6 & 3.8 & 36.2 & 7.3 & 52.0 & 0.0 \\
\hline PI 650142A & 44.3 & 3.1 & 64.9 & 3.2 & 25.0 & 2.8 & 58.4 & 9.6 \\
\hline PI 650142B & 45.0 & 4.2 & 80.6 & 3.1 & 39.9 & 3.2 & 54.9 & 4.9 \\
\hline PI 650143 * & 63.0 & 0.0 & 81.5 & 2.1 & 51.5 & 9.2 & 66.0 & 0.0 \\
\hline PI 650144 & 54.0 & 1.7 & 78.1 & 6.5 & 40.1 & 4.0 & 69.9 & 3.2 \\
\hline PI 650145 & 49.8 & 1.6 & 82.1 & 4.9 & 37.4 & 3.4 & 72.0 & 0.0 \\
\hline PI 650146 & 54.2 & 1.8 & 82.6 & 9.2 & 45.8 & 9.9 & 72.0 & 0.0 \\
\hline PI 650147 & 52.6 & 2.2 & 82.3 & 5.9 & 37.8 & 8.5 & 71.4 & 1.3 \\
\hline PI 650148 & 56.8 & 4.1 & 78.8 & 6.2 & 34.8 & 5.4 & 71.4 & 1.3 \\
\hline PI 650149 & 58.0 & 0.0 & 79.3 & 8.3 & 32.2 & 0.8 & 72.0 & 0.0 \\
\hline PI 650150 & 49.8 & 1.6 & 78.6 & 6.3 & 33.6 & 4.7 & 72.0 & 0.0 \\
\hline PI 650151 & 49.2 & 1.6 & 88.3 & 5.1 & 32.8 & 2.5 & 62.0 & 0.0 \\
\hline PI 650152* & 57.4 & 3.1 & 81.6 & 8.3 & 38.2 & 14.7 & 66.0 & 0.0 \\
\hline PI 650153 & 44.0 & 0.0 & 75.6 & 5.4 & 11.4 & 1.7 & 72.0 & 0.0 \\
\hline PI 650154 & 48.6 & 1.3 & 84.6 & 6.6 & 29.4 & 0.9 & 72.0 & 0.0 \\
\hline PI $650155^{*}$ & 51.4 & 1.3 & 73.4 & 4.4 & 47.6 & 4.6 & 66.0 & 0.0 \\
\hline PI 650156 & 50.5 & 2.1 & 76.5 & 8.2 & 32.5 & 8.0 & 68.1 & 5.2 \\
\hline PI 650157A * & 52.0 & 0.0 & 67.2 & 7.7 & 59.2 & 14.3 & 66.0 & 0.0 \\
\hline
\end{tabular}


Table 4. Cont

\begin{tabular}{ccccccccc}
\hline Accession $^{* *}$ & $\begin{array}{c}\text { Maturity } \\
\text { (days) }\end{array}$ & STDev & $\begin{array}{c}\text { Height } \\
(\mathbf{c m})^{\mathbf{b}}\end{array}$ & STDev & $\begin{array}{c}\text { Width } \\
(\mathbf{c m})^{\mathbf{c}}\end{array}$ & STDev & $\begin{array}{c}\text { Dry } \\
(\text { days) }\end{array}$ & STDev \\
\hline PI 650157B & 49.8 & 5.0 & 75.5 & 5.0 & 30.7 & 10.3 & 61.5 & 6.0 \\
\hline PI 650158A * & 52.0 & 0.0 & 66.2 & 13.8 & 33.4 & 15.5 & 66.0 & 0.0 \\
\hline PI 650158B & 43.8 & 4.0 & 82.6 & 0.9 & 27.4 & 5.2 & 56.0 & 8.9 \\
\hline PI 650159 & 49.4 & 3.1 & 85.2 & 0.8 & 25.4 & 2.7 & 69.2 & 3.8 \\
\hline PI 650163 & 45.4 & 4.8 & 78.6 & 11.1 & 35.8 & 3.9 & 56.0 & 0.0 \\
\hline PI 650164 & 42.0 & 0.0 & 72.0 & 6.8 & 30.0 & 3.5 & 52.0 & 0.0 \\
\hline PI 650165 & 42.0 & 0.0 & 85.4 & 3.2 & 36.6 & 4.8 & 52.0 & 0.0 \\
\hline PI 650166 & 47.9 & 5.0 & 72.4 & 4.1 & 36.6 & 4.6 & 62.0 & 9.5 \\
\hline PI 650167 * & 62.0 & 2.0 & 69.5 & 8.2 & 37.3 & 9.0 & 70.0 & 0.0 \\
\hline PI 650168 * & 52.0 & 0.0 & 69.7 & 1.2 & 61.0 & 3.6 & 66.0 & 0.0 \\
\hline
\end{tabular}

\subsection{Seed Analysis}

Seed characteristics were assessed, and the mean seed weight was seen to range from 0.19 to $1.05 \mathrm{mg}$ per seed (Table 5). Total mean seed weight per plant showed a minimum of $1.45 \mathrm{~g}$ (PI 304270) to a maximum of $5.06 \mathrm{~g}$ (PI 311735) per plant with a median value of $2.6 \mathrm{~g}$ (Table 5). The estimate total seed per plant shows a range from 1604 (PI 650153) to 9225 (PI 650143) seed per plant with a median value of 3328 (Table 5). While the number of seed and total weight of seed per plant did not appear to correlate, the 'winter' cultivars produced greater than average amounts of seed in general. Seed dimensions showed a mean minimum and maximum width of 0.65 and $1.06 \mathrm{~mm}$, respectively (Table 5). The seed mean length ranged from 1.20 to $2.12 \mathrm{~mm}$ with an average of $1.80 \mathrm{~mm}$ (Table 5). The length by width ratio was also compiled and 1.74 to 2.62 and a median value of 2.04 (Table 5).

\subsection{Seed Oil Biochemical Data}

Biochemical analysis of the oil collected shows varying degrees of differences among the 41 accessions of Camelina sativa (L.) Crntz. (Table 6). Oil content (OC) ranged from $23.6 \%$ in PI 650152 to $44.1 \%$ in the PI 650155 accession (Table 6). Fatty acid components included saturated, monounsaturated, and polyunsaturated fatty acids. The most prevalent saturated fatty acid was palmitic acid, ranging from $5.5 \%$ to $9.5 \%$ (Table 6). The most abundant mono-unsaturated fatty acids are oleic acid (C18:1), ranging from $9.1 \%$ to $17.1 \%$, and gondoic acid (C20:1), measured at $10.5 \%$ to $16.4 \%$ (Table 6 ). The most abundant poly-unsaturated fatty acids are linoleic acid (C18:2), from $16.1 \%$ to $28.6 \%$, and linolenic acid (C18:3), measured at $23.5 \%$ to $36.2 \%$ (Table 6). The weight of a thousand seeds (TWS) varied from $0.2006 \mathrm{~g}$ in PI 650167 to $1.0473 \mathrm{~g}$ PI 650153 (Table 6). See Supplemental Table S1 for a complete fatty acid profile of the raw data obtained. To further explore the oilseed yield traits of thousand seed weight (TSW) and total seed weight, one-way ANOVAs were performed to compare accessions. Significant differences between accessions were observed for both TSW (ANOVA, $p<2.2 \times \mathrm{e}^{-16}$ ) and total seed weight (ANOVA, $p<2.2 \times \mathrm{e}^{-16}$ ). TukeyHSD post-hoc analyses were performed for all pair-wise comparisons with results included in Supplemental Table S3 (TSW stats) and Supplemental Table S4 (total seed weight stats). 
Table 5. Seed values.

\begin{tabular}{|c|c|c|c|c|c|c|c|c|c|c|c|}
\hline Accession ${ }^{* *}$ & $\begin{array}{c}\text { Mean Seed } \\
\text { Weight } \\
(\mathrm{mg})^{a}\end{array}$ & STDev & $\begin{array}{l}\text { Mean Total } \\
\text { Weight } \\
\text { (g) }\end{array}$ & STDev & $\begin{array}{l}\text { Est. Total } \\
\text { Seeds per } \\
\text { Plant }^{c}\end{array}$ & $\begin{array}{l}\text { Mean } \\
\text { Width } \\
(\mathrm{mm})\end{array}$ & STDev & $\begin{array}{c}\text { Mean } \\
\text { Length } \\
(\mathrm{mm})\end{array}$ & STDev & $\begin{array}{c}\text { L:W } \\
\text { Ratio }\end{array}$ & STDev \\
\hline PI 258366 & 0.7576 & 0.21 & 3.2091 & 0.19 & 4236 & 0.87 & 0.08 & 1.83 & 0.15 & 2.12 & 0.04 \\
\hline PI 258367 & 0.8624 & 0.11 & 3.1424 & 0.50 & 3644 & 0.99 & 0.03 & 1.97 & 0.03 & 2.00 & 0.03 \\
\hline PI 304269 & 0.6608 & 0.07 & 1.5185 & 0.13 & 2298 & 0.88 & 0.08 & 1.87 & 0.03 & 2.17 & 0.16 \\
\hline PI 304270 & 0.4446 & 0.08 & 1.4478 & 0.27 & 3256 & 0.65 & 0.09 & 1.63 & 0.11 & 2.57 & 0.22 \\
\hline PI 311735 & 1.0219 & 0.13 & 5.0617 & 0.48 & 4953 & 1.06 & 0.05 & 1.95 & 0.06 & 1.85 & 0.11 \\
\hline PI $311736^{*}$ & 0.7931 & 0.13 & 3.7375 & 0.91 & 4712 & 0.90 & 0.05 & 1.70 & 0.01 & 1.90 & 0.11 \\
\hline PI 597833A & 0.7468 & $\mathrm{n} / \mathrm{a}$ & 2.9545 & $\mathrm{n} / \mathrm{a}$ & 3313 & 0.89 & 0.04 & 1.80 & 0.04 & 2.03 & 0.08 \\
\hline PI 597833B & 0.6486 & 0.03 & 3.1004 & 0.52 & 4780 & 0.87 & 0.05 & 1.65 & 0.05 & 1.90 & 0.12 \\
\hline PI 650140 & 0.9130 & 0.07 & 3.0416 & 0.53 & 3331 & 1.02 & 0.02 & 2.12 & 0.05 & 2.09 & 0.07 \\
\hline PI 650141 & 0.5651 & 0.07 & 2.5286 & 0.32 & 4475 & 0.83 & 0.03 & 1.68 & 0.03 & 2.05 & 0.06 \\
\hline PI 650142A & 0.6981 & 0.05 & 2.1806 & 0.24 & 3124 & 0.83 & 0.04 & 1.80 & 0.06 & 2.19 & 0.12 \\
\hline PI 650142B & 0.5487 & 0.02 & 2.4079 & 0.46 & 4388 & 0.76 & 0.00 & 1.73 & 0.03 & 2.30 & 0.03 \\
\hline PI $650143^{*}$ & 0.1916 & 0.01 & 1.7671 & 0.37 & 9225 & 0.70 & 0.01 & 1.31 & 0.00 & 1.87 & 0.04 \\
\hline PI 650144 & 0.6113 & 0.01 & 2.0327 & 0.06 & 3325 & 0.75 & 0.02 & 1.70 & 0.05 & 2.29 & 0.00 \\
\hline PI 650145 & 0.6894 & 0.00 & 1.9309 & 0.22 & 2801 & 0.82 & 0.03 & 1.78 & 0.03 & 2.18 & 0.08 \\
\hline PI 650146 & 0.8062 & 0.06 & 1.8001 & 0.42 & 2233 & 0.81 & 0.05 & 2.04 & 0.06 & 2.55 & 0.18 \\
\hline PI 650153 & 1.0473 & 0.10 & 1.6804 & 0.04 & 1604 & 0.93 & 0.03 & 2.01 & 0.02 & 2.17 & 0.08 \\
\hline PI 650154 & 0.8587 & 0.04 & 2.2814 & 0.18 & 2657 & 0.97 & 0.12 & 1.79 & 0.02 & 1.90 & 0.16 \\
\hline PI $650155^{*}$ & 0.9102 & 0.03 & 4.2544 & 0.99 & 4674 & 0.94 & 0.02 & 1.70 & 0.05 & 1.82 & 0.03 \\
\hline PI 650156 & 0.6941 & 0.09 & 1.8880 & 0.09 & 2720 & 0.81 & 0.04 & 1.78 & 0.09 & 2.21 & 0.11 \\
\hline PI 650157A * & 0.8465 & 0.09 & 3.4222 & 1.70 & 4043 & 0.91 & 0.06 & 1.69 & 0.06 & 1.89 & 0.06 \\
\hline PI 650157B & 0.9095 & 0.07 & 2.3057 & 0.36 & 2535 & 1.00 & 0.09 & 1.97 & 0.06 & 1.99 & 0.21 \\
\hline PI 650158A * & 0.9518 & 0.04 & 1.7637 & 0.55 & 1853 & 0.96 & 0.06 & 1.93 & 0.03 & 2.04 & 0.12 \\
\hline PI 650158B & 0.7851 & 0.08 & 2.0477 & 0.34 & 2608 & 0.94 & 0.07 & 1.86 & 0.07 & 1.98 & 0.17 \\
\hline PI 650159 & 1.0456 & 0.05 & 2.6669 & 0.42 & 2551 & 1.03 & 0.02 & 1.99 & 0.07 & 1.94 & 0.06 \\
\hline PI 650163 & 0.8302 & 0.02 & 3.1996 & 0.20 & 3854 & 0.96 & 0.02 & 1.83 & 0.01 & 1.91 & 0.03 \\
\hline PI 650164 & 0.5916 & 0.14 & 1.9030 & 0.31 & 3217 & 0.89 & 0.09 & 1.77 & 0.08 & 2.02 & 0.16 \\
\hline PI 650165 & 0.7562 & 0.05 & 2.4967 & 0.26 & 3302 & 0.95 & 0.01 & 1.99 & 0.08 & 2.11 & 0.09 \\
\hline PI 650166 & 0.6815 & 0.11 & 2.9354 & 1.06 & 4307 & 0.90 & 0.04 & 1.83 & 0.05 & 2.05 & 0.06 \\
\hline PI $650167^{*}$ & 0.2006 & 0.03 & 1.7985 & 0.75 & 8967 & 0.69 & 0.02 & 1.20 & 0.02 & 1.76 & 0.05 \\
\hline PI $650168^{*}$ & 0.8577 & 0.13 & 4.9498 & 1.17 & 5771 & 0.96 & 0.02 & 1.73 & 0.06 & 1.81 & 0.04 \\
\hline Suneson & 0.7845 & 0.13 & 2.9444 & 0.65 & 3753 & 0.98 & 0.09 & 1.94 & 0.06 & 2.00 & 0.15 \\
\hline
\end{tabular}

* Indicates accessions that required vernalization in order to flower. Also described as 'winter' accessions. ${ }^{* *}$ A sample size $(n)$ of 10 was used per cultivar. See Supplemental Table S2 for specific values. A and B designation were given to accession obtained from the NGRP center that contained more than two unique phenotypes. ${ }^{\mathrm{a}}$ Defined as the average value of 1000 seed total weight divided by 1000 from 10 plants. ${ }^{b}$ Defined as the average total weight of seeds from 10 plants. ${ }^{c}$ Defined as mean total weight divided by the mean seed weight. See Supplemental Tables S1-S4 for specific values. 
Table 6. Oil values.

\begin{tabular}{|c|c|c|c|c|c|c|c|}
\hline Accession ** & $\begin{array}{c}\text { Mean OC } \\
(\%)^{a}\end{array}$ & $\begin{array}{c}\text { 16:0 Palmitic } \\
(\%)\end{array}$ & $\begin{array}{c}18: 1 n-9 \\
\text { Oleic }(\%)\end{array}$ & $\begin{array}{c}\text { 18:2n-6 Linoleic } \\
\text { LA }(\%)\end{array}$ & $\begin{array}{c}18: 3 n-3 \\
\text { ALA (\%) }\end{array}$ & $\begin{array}{c}20: 1 n-9 \\
\text { Gondoic }(\%)\end{array}$ & $\operatorname{TSW}(g)^{b}$ \\
\hline PI 258366 & 36.1 & 6.4 & 15.0 & 25.4 & 26.6 & 12.8 & 0.7576 \\
\hline PI 258367 & 35.5 & 7.6 & 14.6 & 24.8 & 27.9 & 12.6 & 0.8624 \\
\hline PI 304269 & 26.4 & 7.5 & 12.2 & 28.6 & 23.5 & 11.6 & 0.6608 \\
\hline PI 304270 & 26.4 & 6.7 & 14.1 & 23.6 & 28.7 & 13.4 & 0.4446 \\
\hline PI 304271 & 35.6 & 7.0 & 13.8 & 21.2 & 29.4 & 12.9 & 0.6799 \\
\hline PI 311735 & 38.2 & 7.0 & 12.2 & 25.8 & 26.2 & 12.9 & 1.0219 \\
\hline PI 311736 * & 38.3 & 7.9 & 10.7 & 24.8 & 29.5 & 12.0 & 0.7931 \\
\hline PI 597833A & 37.5 & 7.3 & 14.4 & 19.7 & 32.2 & 13.0 & 0.7468 \\
\hline PI 597833B & 36.9 & 7.4 & 13.9 & 19.6 & 33.5 & 12.5 & 0.6486 \\
\hline PI 633192 & 34.7 & 7.1 & 13.7 & 23.1 & 26.7 & 14.4 & 0.6151 \\
\hline PI 633193 * & 33.4 & 6.4 & 14.9 & 18.0 & 33.7 & 13.6 & 0.7759 \\
\hline PI 633194 & 29.6 & 8.3 & 12.2 & 27.4 & 30.1 & 10.5 & 0.6932 \\
\hline PI 650140 & 30.6 & 7.3 & 14.5 & 24.0 & 27.5 & 12.2 & 0.9130 \\
\hline PI 650141 & 33.4 & 9.5 & 15.7 & 28.4 & 30.4 & 10.7 & 0.5651 \\
\hline PI 650142A & 32.7 & 8.6 & 13.8 & 23.7 & 29.7 & 10.9 & 0.6981 \\
\hline PI 650142B & 31.5 & 7.4 & 13.0 & 24.8 & 30.9 & 11.9 & 0.5487 \\
\hline PI 650143 * & 23.9 & 7.5 & 9.8 & 19.7 & 29.2 & 11.4 & 0.1916 \\
\hline PI 650144 & 30.5 & 7.6 & 14.2 & 23.7 & 27.9 & 13.3 & 0.6113 \\
\hline PI 650145 & 34.6 & 7.0 & 13.4 & 18.7 & 36.2 & 12.0 & 0.6894 \\
\hline PI 650146 & 29.3 & 7.4 & 14.1 & 25.6 & 27.8 & 10.7 & 0.8062 \\
\hline PI 650147 & 30.1 & 7.1 & 11.9 & 20.8 & 32.6 & 13.6 & 0.7902 \\
\hline PI 650148 & 26.9 & 8.7 & 10.1 & 21.9 & 33.2 & 12.0 & 0.6605 \\
\hline PI 650149 & 29.2 & 7.5 & 9.1 & 21.3 & 32.0 & 12.8 & 0.5511 \\
\hline PI 650150 & 31.9 & 6.5 & 10.3 & 20.2 & 35.2 & 12.6 & 0.9297 \\
\hline PI 650151 & 29.8 & 6.7 & 14.4 & 21.2 & 32.8 & 12.0 & 0.7199 \\
\hline PI $650152 *$ & 23.6 & 8.7 & 17.1 & 17.3 & 24.9 & 16.4 & 0.3366 \\
\hline PI 650153 & 37.4 & 6.8 & 9.8 & 21.7 & 29.9 & 14.7 & 1.0473 \\
\hline PI 650154 & 33.9 & 6.2 & 10.1 & 19.8 & 33.9 & 13.2 & 0.8587 \\
\hline PI $650155^{*}$ & 44.1 & 5.5 & 14.1 & 18.4 & 34.6 & 13.5 & 0.9102 \\
\hline PI 650156 & 35.9 & 6.4 & 11.0 & 19.1 & 34.3 & 13.5 & 0.6941 \\
\hline PI 650157A* & 37.7 & 5.9 & 13.9 & 17.9 & 32.4 & 15.1 & 0.8465 \\
\hline PI 650157B & 36.4 & 7.0 & 12.6 & 21.2 & 29.6 & 13.3 & 0.9095 \\
\hline PI 650158A* & 35.7 & 7.2 & 13.8 & 16.1 & 32.8 & 14.3 & 0.9518 \\
\hline PI 650158B & 34.8 & 7.1 & 11.4 & 21.6 & 29.2 & 14.5 & 0.7851 \\
\hline PI 650159 & 37.5 & 6.6 & 12.0 & 21.3 & 30.1 & 13.2 & 1.0456 \\
\hline PI 650163 & 39.6 & 6.3 & 13.6 & 20.8 & 32.5 & 13.1 & 0.8302 \\
\hline PI 650164 & 33.6 & 6.9 & 13.3 & 21.6 & 29.0 & 14.3 & 0.5916 \\
\hline PI 650165 & 31.9 & 7.5 & 12.5 & 27.6 & 23.5 & 12.6 & 0.7562 \\
\hline PI 650166 & 44.0 & 7.8 & 12.3 & 27.3 & 24.9 & 11.4 & 0.6815 \\
\hline PI $650167^{*}$ & 25.3 & 6.8 & 12.2 & 21.4 & 29.6 & 12.8 & 0.2006 \\
\hline PI 650168 * & 41.0 & 6.2 & 12.3 & 19.1 & 34.3 & 14.0 & 0.8577 \\
\hline Suneson & 30.7 & 7.5 & 10.6 & 27.0 & 26.0 & 13.0 & 0.7845 \\
\hline
\end{tabular}

*Indicates accessions that required vernalization in order to flower. Also described as 'winter' accessions. ${ }^{* *}$ A sample size $(n)$ of 3 was used per cultivar and mean values presented. See Supplemental Tables S1-S4 for specific values. $A$ and $B$ designation were given to accession obtained from the NGRP center that contained more than two unique phenotypes. ${ }^{a}$ OC_- oil content ${ }^{b}$ TSW-Thousand Seed Weight. 


\subsection{Genetic Analysis}

The camelina accessions were examined for chromosome number. It was determined that $75.6 \%$ of the lines contained the expected $2 n=40$ while unexpectantly $24.3 \%$ contained a $2 n=38$ value. One accession PI 650152 contains a $2 n$ of 26 . Individual lines were also examined for their COT values to provide a unique identifying fingerprint for each cultivar (Table 7). The COT analysis is a technique that provides a way to measure DNA reassociation kinetics and gives a measure of repetitive DNA content per genome [68]. This analysis was used as verification for the predicted $\mathrm{A} / \mathrm{B}$ split in the 4 lines that contained observable differences in phenotypes, and independent from chromosome counts. Table 8 provides a summary of previous tables but specific to the alignment of the A/B accessions. Results show that each $\mathrm{A} / \mathrm{B}$ accession has a unique COT value, chromosome number, vernalization requirement, branch pattern, leaf shape, leaf margin, flowering time, height, and number of days to maturity.

Table 7. Chromosome number and COT value.

\begin{tabular}{|c|c|c|c|}
\hline Accession & Chromosome \# ${ }^{a}$ & COT Value-DNA $(\mathrm{pg} / 2 \mathrm{C})^{b}$ & STDev \\
\hline PI 258366 & 40 & 1.63 & 0.015 \\
\hline PI 258367 & 38 & 1.66 & 0.016 \\
\hline PI 304269 & 40 & 1.58 & 0.008 \\
\hline PI 304270 & 40 & 1.55 & 0.032 \\
\hline PI 304271 & 40 & 1.66 & 0.012 \\
\hline PI 311735 & 38 & 1.64 & 0.012 \\
\hline PI 311736 * & 40 & 1.62 & 0.007 \\
\hline PI 597833A & 40 & 1.41 & 0.017 \\
\hline PI 597833B & 40 & 1.38 & 0.099 \\
\hline PI 633192 & 40 & 1.60 & 0.008 \\
\hline PI 633193 * & 40 & 1.61 & 0.016 \\
\hline PI 633194 & 40 & 1.61 & 0.029 \\
\hline PI 650140 & 40 & 1.62 & 0.022 \\
\hline PI 650141 & 38 & 1.60 & 0.020 \\
\hline PI 650142A & 40 & 1.44 & 0.052 \\
\hline PI 650142B & 40 & 0.58 & 0.017 \\
\hline PI 650143 * & 38 & 1.29 & 0.097 \\
\hline PI 650144 & 38 & 1.25 & 0.094 \\
\hline PI 650145 & 40 & 1.33 & 0.083 \\
\hline PI 650146 & 38 & 1.33 & 0.070 \\
\hline PI 650147 & 40 & 1.37 & 0.027 \\
\hline PI 650148 & 40 & 1.27 & 0.034 \\
\hline PI 650149 & 40 & 1.30 & 0.040 \\
\hline PI 650150 & 40 & 0.98 & 0.072 \\
\hline PI 650151 & 40 & 1.43 & 0.031 \\
\hline PI $650152 *$ & 26 & 1.15 & 0.095 \\
\hline
\end{tabular}


Table 7. Cont

\begin{tabular}{cccc}
\hline Accession & Chromosome \# $^{\mathbf{a}}$ & ${\text { COT Value-DNA }(\mathbf{p g} / \mathbf{2 C})^{\mathbf{b}}}^{\mathbf{c}}$ & STDev \\
\hline PI 650153 & 40 & 1.20 & 0.063 \\
\hline PI 650154 & 40 & 0.97 & 0.088 \\
\hline PI 650155 * & 40 & 1.42 & 0.083 \\
\hline PI 650156 & 40 & 0.83 & 0.093 \\
\hline PI 650157A * & 40 & 1.35 & 0.014 \\
\hline PI 650157B & 38 & 1.23 & 0.045 \\
\hline PI 650158A * & 40 & 1.35 & 0.025 \\
\hline PI 650158B & 40 & 0.97 & 0.043 \\
\hline PI 650159 & 40 & 1.46 & 0.015 \\
\hline PI 650163 & 40 & 1.66 & 0.027 \\
\hline PI 650164 & 38 & 1.62 & 0.020 \\
\hline PI 650165 & 40 & 1.58 & 0.018 \\
\hline PI 650166 & 40 & 1.67 & 0.023 \\
\hline PI 650167 * & 38 & 1.61 & 0.036 \\
\hline PI 650168* & 38 & 1.48 & 0.015 \\
\hline Suneson & 40 & 1.14 & 0.063
\end{tabular}

* Indicates accessions that required vernalization in order to flower. Also described as 'winter' accessions. A and B designation were given to accession obtained from the NGRP center that contained more than two unique phenotypes. ${ }^{a}$ Date present as a $2 n$ value. Sampling size ranged from 3-33 to determine chromosome counts. See Supplemental Table S2 for details. ${ }^{b}$ COT value is defined as DNA reassociation kinetics and gives a measure of repetitive DNA content per genome. Sampling size was 1000 nuclei. 
Table 8. A/B cultivar analysis.

\begin{tabular}{|c|c|c|c|c|c|c|c|c|c|c|c|}
\hline Accession ${ }^{* *}$ & $\begin{array}{c}\mathrm{COT} \\
(\mathrm{pg} / 2 \mathrm{C})^{\mathrm{a}}\end{array}$ & Chrom \# ${ }^{b}$ & Vernalize $^{c}$ & $\begin{array}{l}\text { Branch } \\
\text { Pattern }{ }^{d}\end{array}$ & Leaf Shape & $\begin{array}{c}\text { Leaf } \\
\text { Margin }\end{array}$ & $\begin{array}{l}\text { Flowering } \\
\text { (Days) }^{\mathrm{e}}\end{array}$ & $\begin{array}{c}\text { Height at } \\
\text { Flowering }(\mathrm{cm}) \mathrm{f}\end{array}$ & $\begin{array}{l}\text { Florets in Initial } \\
\text { Flower } \mathrm{g}\end{array}$ & $\begin{array}{l}\text { Maturity } \\
\text { (days) h }\end{array}$ & $\begin{array}{c}\text { Height at } \\
\text { Maturity }(\mathrm{cm})^{i}\end{array}$ \\
\hline PI 597833A & 1.41 & 40 & $\mathrm{~N}$ & $\mathrm{~W}$ & linear & serrate & 33.0 & 38.8 & 10.3 & 50.3 & 67.2 \\
\hline PI 597833B & 1.38 & 40 & $\mathrm{~N}$ & $\mathrm{~W}$ & lanceolate & spiny & 31.3 & 46.8 & 9.6 & 48.3 & 80.4 \\
\hline PI 650142A & 1.44 & 40 & $\mathrm{~N}$ & $\mathrm{~W}$ & linear & serrate & 31.3 & 38.5 & 12.0 & 44.3 & 64.9 \\
\hline PI 650142B & 0.58 & 40 & $\mathrm{~N}$ & $\mathrm{~W}$ & lanceolate & spiny & 33.3 & 45.6 & 12.4 & 45.0 & 80.6 \\
\hline PI 650157A * & 1.35 & 40 & $\mathrm{Y}$ & $x$ & subulate & serrate & 28.6 & 28.6 & 11.0 & 52.0 & 67.2 \\
\hline PI 650157B & 1.23 & 38 & $\mathrm{~N}$ & $\mathrm{Z}$ & subulate & smooth & 33.5 & 40.7 & 10.0 & 49.8 & 75.5 \\
\hline PI 650158A* & 1.35 & 40 & $\mathrm{Y}$ & $\mathrm{W}$ & lanceolate & spiny & 31.0 & 36.6 & 10.6 & 52.0 & 66.2 \\
\hline PI 650158B & 0.97 & 40 & $\mathrm{~N}$ & W & lanceolate & smooth & 31.2 & 47.2 & 9.4 & 43.8 & 82.6 \\
\hline
\end{tabular}

* Indicates accessions that required vernalization in order to flower. Also described as 'winter' accessions. ** A sample size (n) of 3-12 was used per cultivar. See Supplemental Table S2 for specific values. A and B designation were given to accession obtained from the NGRP center that contained more than two unique phenotypes. ${ }^{a}$ COT value is defined as DNA reassociation kinetics and gives a measure of repetitive DNA content per genome ${ }^{b}$ Sampling size ranged from 3-33 to determine chromosome counts. See Supplemental Table S2 for reassociation kinetics and gives a measure of repetitive DNA content per genome ${ }^{b}$ Sampling size ranged from $3-33$ to determine chromosome counts. See Supplemental Table $\mathrm{S} 2$ for
details. $^{c}$ Defined as requirement to keep seed for 8 weeks at $4{ }^{\circ} \mathrm{C}$ before being able bolt or flower. ${ }^{d} \mathrm{~W}$-Top Heavy branching often seen with secondary branching; $X-$ Branched length of main stem with few secondary branches; Y-Tiller where all branches originate from the base and contain few secondary branching; $Z-C h a o s$ where branches are seen originating everywhere and a dominant stem was not observed. ${ }^{\text {e }}$ Defined as time when greater than $50 \%$ of all floret within the initial composite flower are open. ${ }^{\mathrm{f}}$ Maximum height of plant as determined at initial/primary flowering. ${ }^{\mathrm{g}}$ Number of florets seen in the initial composite flower on the initial floral bolt. ${ }^{\mathrm{h}}$ Defined as time when greater than $50 \%$ of all flowers are open and seed set has begun. ${ }^{\mathrm{i}}$ Maximum height of plant at maturity. 


\subsection{Advances in Biotechnology}

Biotechnological enhancements have been achieved to complement the traditional cultivar improvement efforts of breeding and mutagenesis. To add to and improve upon these biotechnology efforts, our lab investigated the use of positive as well as negative selection marker genes to facilitate techniques such as RMCE and GAANTRY [56,57] for metabolic engineering.

We chose to investigate the positive marker genes bar, [41], hptII [69], nptII [70] and sulI [71] for use in camelina selection, as they have all been shown to work in Arabidopsis. The optimal range of selective agents to inhibit camelina growth was determined for each (Supplemental Figures S1-S5). The negative selective marker gene, $\operatorname{cod} A$, was also investigated for monitoring DNA excision events [72,73]. The $\operatorname{cod} A$ gene required a kill curve be determined for both 5-fluorocytosine (5FC) and 5-fluorouracil (5FU (Supplemental Figure S6). In the presence of a functional $\operatorname{cod} A$ gene the nontoxic 5FC is converted to it toxic form 5FU. The toxin 5FU is a DNA chain-terminating compound that will stunt or kill germinating seed at very low concentrations.

Employing the Lu and Kang [41] method, a series of binary vectors were transformed into the camelina cultivar Suneson (Supplemental Figure S1). These vectors were designed with a positive/negative marker cassette flanked by recombinase recognition sites for use as RMCE founder lines [56] and capable of gene stacking. During the hygromycin (hptII) selection trial, 13 transformed lines were recovered. All lines contained a high T-DNA copy number (4 or more), as determined by Southern blot analysis (SBA) (Figure 3A). Plants under selection appeared sickly and were difficult to identify against background (wild-type) growth. However, plants did recover once transferred to soil. Glufosinate (bar) selection identified 14 plants that showed both low and multiple copy lines by SBA (Figure 3A). Identified plants appeared healthy under selection. Sulfadiazine revealed one line from initial trials; it was determined to be a 2 copy T-DNA insertion event by SBA and the plant appeared healthy (Figure 3A). The final positive selection marker tested was nptII. While a kill curve range was determined for the antibiotics kanamycin and G418 (Geneticin) (Supplemental Figure S2), use of the nptII selection gene, driven by the double enhanced 355 promoter (pCTAG-GCN), was only sufficient to produce a single resistance transgenic camelina from $\sim 20,000$ seeds screened.

To determine if this was a failure of the marker or poor rates of transformation, a second binary vector previously used for transformation, pCTAGV-KCN3 [72] was used. Seed selection was split onto either kanamycin selection or DsRed (visual) selection for germinating seedlings. The fluorescent DsRed selection marker under a constitutive promoter has previously been used to identify transformed seed based on fluorescence [41]. Five plants were obtained using visual DsRed selection, while zero plants were obtained from kanamycin selection of $\sim 1000$ plated seeds.

For $\operatorname{cod} A$ negative selection testing, $\mathrm{T}_{2}$ seeds from the aforementioned hygromycin, sulfadiazine and glufosinate positive selection studies were germinated in the presence of $500 \mathrm{mg} \mathrm{L}^{-1}$ 5FC. Results can be seen in Figure 3B, C and indicate that in the presence of a functional codA gene and the selective agent 5FC, camelina plants appear to grow yellow and stunted as compared to a null segregating sibling from the same transformation event that appears green and robust.

In an attempt to determine whether any of the 41 lines investigated were viable for biotechnological manipulation we chose the line PI 311735 (due to its large seed and high oil content) for transformation using the pCTAG-GBC binary vector and glufosinate (bar) selection. Rates of transformation were similar to the control accession Suneson for production of transgenic plants through the floral dip method described [41]. While not overly effective we were able to obtain transgenic camelina plants with an efficiency of $0.6 \%$ using bar selection. PCR was used to provide molecular verification of transgenic camelina obtained from the PI 311735 and Suneson lines (Supplemental Figure S7). 
A

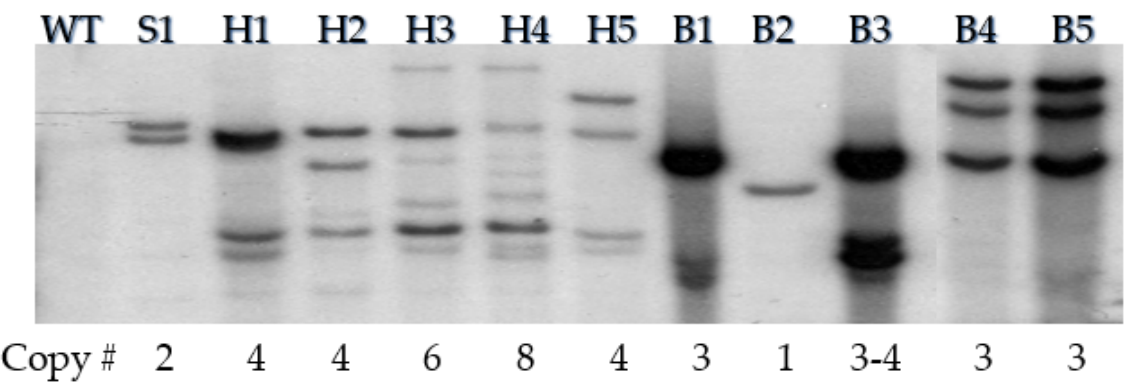

B

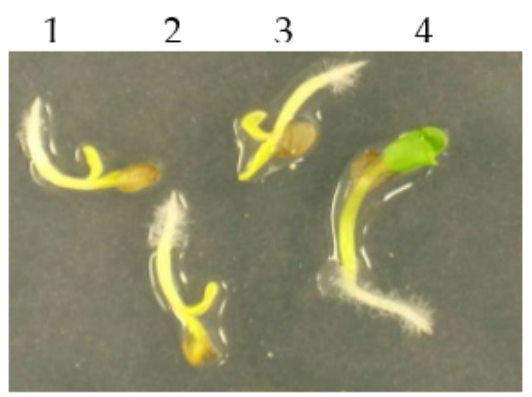

C

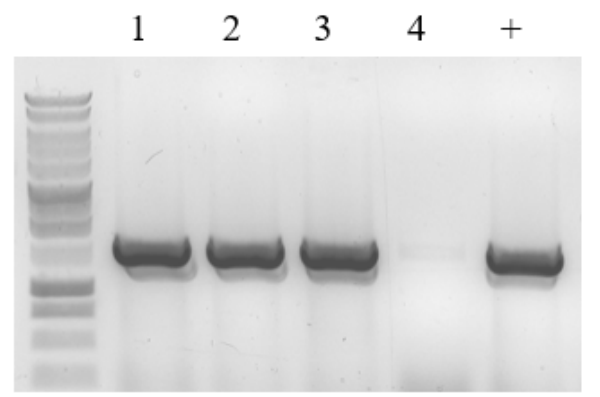

Figure 3. Biotechnology. (A) Southern blot analysis of camelina obtained from selection on sulfadiazine $150 \mathrm{mg} / \mathrm{L}$ (S1), hygromycin $30 \mathrm{mg} / \mathrm{L}$ (H1-5) and Basta $25 \mathrm{mg} / \mathrm{L}$ (B1-5). (B) Germination experiment of CTAG-35HC \#2 $\mathrm{T}_{1}$ Camelina seeds on MS media containing $500 \mathrm{mg} / \mathrm{L}$ of 5-FC. Seedlings 1, 2, and 3 had a yellow, stunted phenotype, while seedling 4 was green and had healthy growth. (C) DNA was extracted from seedlings and analyzed using PCR with primers specific for the $\operatorname{cod} A$ transgene.

\section{Discussion}

The genus Camelina is composed of 11 species [74] but as of 2011, when seeds for this study were obtained, only five species: (C. sativa, C. microcarpa, C. rumelica, C. alyssum, and C. hispida) were present in the germplasm of the IPK (Institute of Plant Genetics and Crop Plant Research, Gatersleben, Germany) and the USDA-NGRP repositories. Among them, only C. sativa and C. microcarpa are cultivated. Within C. sativa, three different subspecies, ssp. pilosa, ssp. sativa and ssp. foetida, have been described [75].

Chromosome analysis of the 41 accessions (Table 6) displayed an interesting set of results. First, a single accession PI 650152 was found to contain an $n=13$ and may have been mis-classified, although it appears to display phenotypic characteristics similar to Camelina sativa (L.) Crntz. Next, a split in chromosome number was obtained and it appears that $75.6 \%(31 / 41)$ lines have the predicted $n=20$ chromosome number while $24.3 \%(10 / 41)$ have an $n=19$. Previous results indicate that Camelina sativa (L.) Crntz. is an allohexaploid plant and in 2006, the Snowdon lab [50] demonstrated through the use of 157 AFLP marker linkage map and 3 Brassica SSR markers that the chromosome number of camelina was $n=20$. These results were confirmed when the genome was sequenced, and a genome size of $750 \mathrm{Mbp}$ was reported [49,76] for two different cultivars-accessions used were unpublished. It appears that triplication of the camelina genome occurred through whole genome duplication by either autopolyploidization or allopolyploidization. Though an autopolyploidy event triplicating a single diploid genome would result in an autohexaploid with a haploid genome of $n=18$, 21 , or 24 chromosomes depending on a starting genome chromosome count of $n=6,7$, or 8 , respectively. However, camelina has reported chromosome counts of $n=6,7,10,13,18,19,20[76,77]$. 
Based on previous reports, $n=20$ appeared to be the most common value and agrees with the recent AFLP marker linkage map and sequencing data. However, an $n=20$ chromosome count would be difficult (although not impossible) to achieve through a single event triplicating a diploid genome. Triplication of the camelina genome from two allopolyploidy events, resulting in first a tetraploid followed by a second polyploid mating to produce a hexaploid, similar to the origin of cultivated 6-row wheat, is more likely. From previous research it appears that hybridization via outcrossing to related species is possible within the Brassicaceae family [78,79]. Taking into consideration the reported chromosome counts of various camelina related species, an initial allopolyploidy hybrid cross resulting from two diploid parental species where one was $n=6$ and the other $n=7$ could contribute to the production of a tetraploid genome with 13 chromosomes and would explain cultivar PI 650152. This is possible considering that related species C. laxa and Camelina spp. have $n=6$, C. hispida has $n=7$, and C. rumelica has $n=13$ chromosome counts [80]. Following this logic, a second allopolyploidy hybridization event producing hexaploid progeny could be achieved by mating the tetraploid progeny $(n=13)$ with either of the two starting parental lines where a $13+6$ cross would result in an $n=19$ and a $13+7$ event would result an $n=20$ chromosome count. These hypothetical crosses would explain the varied chromosome counts documented in numerous camelina publications. The hypothetical crossing scenario is further supported by a recent publication [81] where the genetic diversity of the camelina genus was assessed across 54 accessions representing five species through RADseq, ITS sequencing, and flow cytometry. Results of the investigation infer that an $(n=6+7+7)$ hybridization is possible. The allopolyploid hypothesis is also supported by the observation that C. sativa demonstrates diploid inheritance [41,48], as would be expected for an allopolyploid [82]. A hexaploid C. sativa could also be derived from the combination of an autotetraploid and a diploid species if, in the autopolyploid genome, homologous chromosomes differentiated, so the subsequent chromosome-specific pairing mimicked an allopolyploid genome in its diploid inheritance patterns [82]. Regardless of its evolutionary path, the $C$. sativa genome appears organized in three redundant and differentiated copies and can be formally considered to be an allohexaploid. Our results support previous research and add to it that two hexaploid combinations exist within the known NGRP accessions $n=6+7+7(20)$ and $n=6+7+6(19)$.

From an agronomic point of view, C. sativa ssp. sativa and Camelina sativa ssp. Pilosa, sometimes termed 'winter' camelina, seem to be the most promising subspecies for abundant seed production, where 8 of the 9 cultivars examined had an above average seed count (4043 to 9225) when compared to the accessions as a whole (3328), see Table 7 . These winter camelina subspecies are usually sown in autumn, since they require vernalization in order to attain stem elongation and subsequent flowering, while Camelina sativa ssp. foetida (aka., 'spring' Camelina) does not require vernalization and can be sown in both autumn and spring. All cultivars examine had an early emergence phenotype (2 days on average) that appears characteristic of the species in general, see Table 1. Bolting or time to flowering and time to dry are agronomic traits of importance, with the NGRP accessions ranging from 18 to $35^{\prime}$ days and 52 to 72 days, respectively, see Tables 3 and 4 . From data observed rapid maturing and drying accessions appear to be PI 650145 and PI 650146. These lines may offer an opportunity to cultivate rapid cycling genotypes for double cropping utilization.

Total seed weight for the various accessions ranged from a minimum of $1.45 \mathrm{~g}$ (PI 304270) to a maximum of $5.06 \mathrm{~g}$ (PI 311735). The oil content in dry weight seeds ranges between 23.6\% (PI 650152) and $44.1 \%$ (PI650115) and consists of approximately $54 \%$ polyunsaturated, $34 \%$ monounsaturated, and $12 \%$ saturated fatty acids. The most abundant poly-unsaturated fatty acids are linoleic acid (C18:2), ranging from $10.5 \%$ to $16.4 \%$, and linolenic acid (C18:3), ranging from $23.5 \%$ to $36.2 \%$. The mono-unsaturated erucic acid (C22:1) is of importance for feed, with a maximum value of $<2 \%$ allowed. Only one line, PI 650140, falls within this parameter at 1.83\%, (Supplemental Table S1) and potentially provides useful breeding stock for improvement to camelina as an animal feed. Taken as a whole accession PI 311735 appears to provide an excellent set of agronomic traits for an oil seed crop. The cultivar has one of the earliest bolting times, at 18 days and a better than average drying time at 
60.8 days. However, its most outstanding characteristics are its large total seed weight at 5.06 gram per plant and its above average oil content, measured at 38.2\%. Accession PI 311735 was further tested for potential genetic manipulation through the agrobacterium 'flora dip' transformation technique in an attempt to produce a transgenic plant. Our lab successfully produced six transgenic plants as verified by seedling selection on glufosinate and confirmation by PCR. These results validate that this accession could undergo genetic modification.

From our examination the selectable marker bar (glufosinate) still appears to be the best selection system available for camelina genome modification. However, our research indicates that sulI (sulfadiazine) may be a viable option. Unfortunately, a more thorough study will be needed to validate this claim. The negative selectable maker gene $\operatorname{cod} A$ was successful in inhibiting seedling growth in the presence of 5FC. Seedlings could even be rescued from the 5FC selection plate and grown to produce viable plants (data not shown). This will provide a useful selection tool for monitoring DNA excision events for techniques such and CRISRP and RMCE.

\section{Materials and Methods}

Throughout the phenotypic descriptions, growth stages of camelina sativa presented are subtitled according to the two-digit BBCH scale [67] in the text. In the present investigation, plants were grown and evaluated under non-crowded greenhouse conditions in the California Bay area and therefore may not perfectly reflect more stressful field conditions in other parts of the country.

Table 9 presents accessions used listed according to the NGRP center designation, countries of origin and other names associated with these cultivars.

Table 9. NGRP center designations.

\begin{tabular}{|c|c|c|c|c|c|}
\hline Accession & Lot\# & $\begin{array}{l}\text { Scientific } \\
\text { Name }\end{array}$ & Other Name(s) & $\begin{array}{l}\text { Country of } \\
\text { Origin }\end{array}$ & Donor \\
\hline PI 258366 & 06ncai01 & Camelina sativa & VNIIMK 17 & Soviet Union & NGRP \\
\hline PI 304269 & 06ncai02 & Camelina sativa & No. 402 & Sweden & NGRP \\
\hline PI 304270 & 97ncei01 & Camelina sativa & No. 403 & Sweden & NGRP \\
\hline PI 311735 & 06ncai01 & Camelina sativa & Ames 1043 & Poland & NGRP \\
\hline PI $311736^{*}$ & 94ncai01 & Camelina sativa & Ames 1042 & Poland & NGRP \\
\hline PI 597833A & 94ncai01 & Camelina sativa & Ames 21330; 163-2073-72 & Denmark & NGRP \\
\hline PI 597833B & 94ncai01 & Camelina sativa & Ames 21330; 163-2073-72 & Denmark & NGRP \\
\hline PI 650140 & 97ncai01 & Camelina sativa & Ames 22986; CR 1673/90d; Came & Germany & NGRP \\
\hline PI 650141 & 04ncai01 & Camelina sativa & Ames 24253; NU 52279; & $\begin{array}{l}\text { United States, } \\
\text { Minnesota }\end{array}$ & NGRP \\
\hline PI 650142A & 02ncai01 & Camelina sativa & Ames 26665; G 31712; CS-163-2073-72 & Denmark & NGRP \\
\hline PI 650142B & 02ncai01 & Camelina sativa & Ames 26665; G 31712; CS-163-2073-72 & Denmark & NGRP \\
\hline PI 650143 * & 06ncai01 & Camelina sativa & Ames 26666; G 31713; CS-CR00 & Germany & NGRP \\
\hline PI 650144 & 02ncni01 & Camelina sativa & Ames 26667; G 31714; CS-CR1670; Boha & Denmark & NGRP \\
\hline PI 650145 & 06ncai01 & Camelina sativa & Ames 26668; G 31715; CS-CR1671; BRSCHW 28347 & $\begin{array}{c}\text { Germany, } \\
\text { Mecklenburg-W.P. }\end{array}$ & NGRP \\
\hline
\end{tabular}


Table 9. Cont.

\begin{tabular}{|c|c|c|c|c|c|}
\hline Accession & Lot\# & $\begin{array}{l}\text { Scientific } \\
\text { Name }\end{array}$ & Other Name(s) & $\begin{array}{c}\text { Country of } \\
\text { Origin }\end{array}$ & Donor \\
\hline PI 650150 & 02ncai01 & Camelina sativa & Ames 26673; G 31720; CS-CR1676; Hoga & Denmark & NGRP \\
\hline PI $650152 *$ & 08ncai01 & Camelina $s p$ & Ames 26675; G 31722; CPS-CAM23 & Germany & NGRP \\
\hline PI 650153 & 02ncai01 & Camelina sativa & Ames 26676; G 31723; CPS-CAM10 & Soviet Union & NGRP \\
\hline PI $650155^{*}$ & 02ncai01 & Camelina sativa & Ames 26678; CSS-CAM27; G 31725; & Poland & NGRP \\
\hline PI 650156 & 02ncni01 & Camelina sativa & Ames 26679; CSS-CAM29; G 31726 & Soviet Union & NGRP \\
\hline PI 650157A* & 02ncai01 & Camelina sativa & Ames 26680; CSS-CAM30; G 31727 & Soviet Union & NGRP \\
\hline PI 650157B & 02ncai01 & Camelina sativa & Ames 26680; CSS-CAM30; G 31727; & Soviet Union & NGRP \\
\hline PI 650158A* & 02ncai01 & Camelina sativa & Ames 26681; CSS-CAM31; G 31728 & Poland & NGRP \\
\hline PI 650163 & 02ncni01 & Camelina sativa & Ames 26686; CSS-CAM37; G 31733 & Soviet Union & NGRP \\
\hline PI 650164 & 02ncni01 & Camelina sativa & Ames 26687; CSS-CAM38; G 31734 & Austria & NGRP \\
\hline PI 650165 & 02ncni01 & Camelina sativa & Ames 26688; CSS-CAM7; G 37135 & Soviet Union & NGRP \\
\hline PI 650166 & 02ncni01 & Camelina sativa & Ames 26689; CSS-CAM8; G 31736 & Soviet Union & NGRP \\
\hline PI $650167 *$ & 08ncai01 & Camelina sativa & Ames 27286; Index Seminum 144 & Poland, Przemysl & NGRP \\
\hline PI 650168 * & 08ncai01 & Camelina sativa & Ames 28372; NE2006-01 & $\begin{array}{c}\text { United States, } \\
\text { Nebraska }\end{array}$ & NGRP \\
\hline Suneson ${ }^{\mathrm{a}}$ & & Camelina sativa & Ames 1043; Ames 26665; “Calena” A3U7761 & $\begin{array}{c}\text { United States, } \\
\text { Montana }\end{array}$ & U of Mont \\
\hline
\end{tabular}

* Indicates accessions that required vernalization in order to flower. Also described as 'winter' accessions. A and B designation were given to accession obtained from the NGRP center that contained more than two unique phenotypes. Accession PI 650152 was designated Camelina sp. due to its unique chromosome count described later. a Suneson is a commonly used accession in the US not provided by NGRP. Suneson or Montana 0305 was release by Montana State University as a mid-season, average-yield line that is high in $\alpha$-linolenic acid (C18:3n3). It was included in this study for its known ability to be transformed by the floral dip method.

\subsection{Early Emergence Studies}

After seeds were initially harvested, they were dried at $30{ }^{\circ} \mathrm{C}$ for one week and then weighed. To test to rate of germination, seed were imbibed on sterile $3 \mathrm{~mm}$ Whatman paper saturated with purified MQ water. It was observed that all viable seed for every cultivar formed a mucosal/gelatinous coat within the first 5 minutes of being imbibed. Seeds were placed on growth racks at $24^{\circ} \mathrm{C}$ and observed for radical emergence. Vernalization was required for 9 of the 41 cultivars and these are indicated by asterisks at the end of their accession number (Table 1). These 'winter' types remained at the rosette stage indefinitely under greenhouse conditions, if not first cold-treated for the required vernalization time period to induce its bolting capacity. Therefore, all accessions were sown on moist soil and kept in the dark at $4{ }^{\circ} \mathrm{C}$ for 14 days.

\subsection{Greenhouse Phenotype Studies}

Seeds were imbibed in water for $1 \mathrm{~h}$ prior to sowing in soil. For each accession, 5 seeds were sown per pot on the surface of damped soil treated with Gnatrol (3-12 pots were sowed depending on accession viability). All accessions once sown were kept in the dark at $4{ }^{\circ} \mathrm{C}$ for 14 days prior to placing in the greenhouse. Greenhouse growth conditions consisted of $18 \mathrm{~h}$ light, $6 \mathrm{~h}$ dark cycles at $26^{\circ} \mathrm{C}$ and $24{ }^{\circ} \mathrm{C}$, respectively. Whole plant measurements were taken every $3-4$ days. Seeds were harvested when dry. Three to twelve pots were planted per accession depending on seed viability. 


\subsection{Seed Quality Traits}

The weight of thousand seeds (TSW—-thousand seed weight) was determined by measuring three replicates of thousand seeds each. Seeds were counted manually and weighed to the nearest $0.1 \mathrm{mg}$. Seeds were measured manually to the nearest $0.01 \mathrm{~mm}$.

\subsection{Seed Quality Trait Statistics}

ANOVA and TukeyHSD statistical analyses of oilseed yield traits were performed in R (version 3.6.3) using aov() and TukeyHSD() functions. Prior to analyses, accessions with mixed traits (designated with $\mathrm{A} / \mathrm{B}$ ) and/or missing values were removed for a balanced design with $n=3$ measurements per accession. Residual plots and Shapiro-Wilk normality tests were done to inspect ANOVA assumptions of normality and homogeneity of variance, and a square root transformation was implemented for total seed weight.

\subsection{Oil Extraction and Weight to Volume Determination}

Oil content for Camelina seeds (sample size 0.4-0.6 g, weighed to four decimal places) was determined non-destructively using a Bruker seed analyzer (Fremont, CA, USA) calibrated for Camelina, with each determination done in triplicate. From each Camelina accession, three samples $(0.5 \mathrm{~g})$ of dry seeds were ground with hexane $(0.1 \% \mathrm{BHA})$ in a glass homogenizer $\left(1.5 \mathrm{~mL} \mathrm{~g}^{-1}\right.$ tissue) and poured into a $16 \times 100 \mathrm{~mm}$ screw cap tube with a Teflon-lined screw cap. The solution was agitated for $30 \mathrm{~min}$. The extract was then centrifuged with desktop Dynac centrifuge (Becton, Dickinson and Company) for $10 \mathrm{~min}$ at $1000 \mathrm{rpm}(140 \mathrm{~g})$ and the supernatant collected. The extraction procedure was repeated on the sediment. The hexane was evaporated under nitrogen and the residue dissolved in $1 \mathrm{~mL}$ of hexane $(0.1 \% \mathrm{BHA})$ for further analysis.

\subsection{Camelina Chromosome Squashes}

For chromosome counts, 15 seed of each accession were germinated on moist filter paper in a $27^{\circ} \mathrm{C}$ growth chamber. Root tips were collected 5 days after germinated and were pretreated

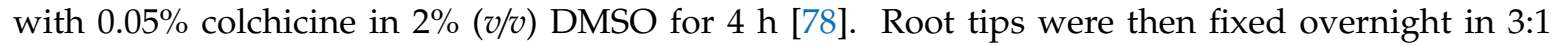
ethanol/glacial acetic acid. Slide preparations were made by digesting root tips for 30- to 60-min with $0.05 \mathrm{~g} \mathrm{~L}^{-1}$ Onuzuka R-10 cellulase and $0.01 \mathrm{~g} \mathrm{~L}^{-1}$ pectolyase $\mathrm{Y}-23$ (Phytotechnology Labs) in $0.01 \mathrm{M}$ citrate buffer $\mathrm{pH} 4.8$ prior to. Digestion time varied according to the thickness and degree of lignification of the roots. Squashes were prepared according to Kirov et al., [83] and counts and were mounted in VECTASHIELD (Vector Laboratories) antifade mounting medium with DAPI (4,6-diamidino-2-phenylindole). Slide preparations were visualized under an Olympus BX51 fluorescent microscope.

\subsection{COT Value Determination}

The COT analysis is a technique that provides a way to measure DNA reassociation kinetics and gives a measure of repetitive DNA content per genome [54]. The procedure used to analyze nuclear DNA content in plant cells was modified from [84]. Briefly, the procedure consists of preparing suspensions of intact nuclei by chopping of $50 \mathrm{mg}$ plant tissues in $\mathrm{MgSO}_{4}$ buffer mixed with DNA standards and stained with propidium iodide (PI) in a solution containing DNAase-free-RNAase. Fluorescence intensities of the stained nuclei are measured by a flow cytometer. Values for nuclear DNA content are estimated by comparing fluorescence intensities of the nuclei of the test population with those of an appropriate internal DNA standard that is included with the tissue being tested. Nuclei from Arabidopsis thaliana $(0.36 \mathrm{pg} / 2 \mathrm{C})$ was used as the internal standard. The pellet is suspended by vortexing vigorously in $0.5 \mathrm{~mL}$ solution containing $10 \mathrm{mM} \mathrm{MgSO}_{4} .7 \mathrm{H}_{2} \mathrm{O}, 50 \mathrm{mM} \mathrm{KCl}, 5 \mathrm{mM}$ Hepes, $\mathrm{pH}$ 8.0, $3 \mathrm{mM}$ dithiothreitol, $0.1 \mathrm{mg} / \mathrm{mL}$ propidium iodide, $1.5 \mathrm{mg} / \mathrm{mL}$ DNAse free RNAse (Rhoche, Indionapolis, IN, USA), and $0.25 \%$ Triton X-100. The suspended nuclei are withdrawn using a pipettor, 
filtered through $30-\mu \mathrm{m}$ nylon mesh, and incubated at $37^{\circ} \mathrm{C}$ for $30 \mathrm{~min}$ before flow cytometric analysis. Suspensions of sample nuclei is spiked with suspension of standard nuclei (prepared in above solution) and analyzed with a FACScalibur flow cytometer (Becton-Dickinson, San Jose, CA, USA). For each measurement, the propidium iodide fluorescence area signals (FL2-A) from 1000 nuclei are collected and analyzed by CellQuest software (Becton-Dickinson, San Jose, CA, USA) on a Macintosh computer. The mean position of the G0/G1 nuclei peak of the sample and the internal standard are determined by CellQuest software. The mean nuclear DNA content of each plant sample, measured in picograms, are based on 1000 scanned nuclei.

\subsection{Plant Transformation}

Modified from Lu and Kang [41]. From a single colony of Agrobacterium, grow $3 \mathrm{~mL}$ starter culture overnight at $28^{\circ} \mathrm{C}$. Inoculate $300 \mathrm{~mL}$ large-scale culture with starter culture and grow overnight at $28^{\circ} \mathrm{C}$ with agitation. Collect cells by centrifugation, then suspend cells in transformation media (0.5X MS salts, 1X Gamborg vitamins, $50 \mathrm{~g} / \mathrm{L}$ Sucrose, $0.01 \mathrm{mg} / \mathrm{L} \mathrm{BAP,} 20 \mathrm{mg} / \mathrm{L}$ acetosyringone, $0.5 \mathrm{~mL} / \mathrm{L}$ Silwet 77). Submerge the initial Camelina inflorescences into Agrobacterium suspension, and then swirl flowers gently in solution and vacuum infiltrated for $3 \mathrm{~min}$. Wrap flowers in plastic wrap and store overnight in darkened room. Next day, unwrap flowers, and return plants to greenhouse to mature. Collect seed, then select transformants as described.

\subsection{Plant Selection}

Antibiotics, including kanamycin, G418 (nptII), hygromycin (hptII), and sulfadiazine (sulI), and the herbicide glufosinate (bar), to determine an effective concentration for routine for a floral dip protocol and positive seed selection. In addition, 5-fluorocytocine (5FC) and 5-fluorouracil (5FU) were investigated for negative selection. It was determined that a concentration of $200 \mathrm{mg} \mathrm{L}^{-1}$ for kanamycin, $30 \mathrm{mg} \mathrm{L}^{-1}$ for $\mathrm{G} 418,30 \mathrm{mg} \mathrm{L}^{-1}$ for hygromycin, $150 \mathrm{mg} \mathrm{L}^{-1}$ for sulfadiazine and $15 \mathrm{mg} \mathrm{L}^{-1}$ for glufosinate ammonium were effective at inhibiting growth of seedlings (Supplemental Figures S1-S5). Camelina was tolerant of 5FC up to $1000 \mathrm{mg} \mathrm{L}^{-1}$ but sensitive for 5FU at concentrations as low as $20 \mathrm{mg} \mathrm{L}^{-1}$ (Supplemental Figure S6).

\section{Conclusions}

From our phenotypic evaluation of the 41 camelina accessions obtained from National Genetic Resources Program (NGRP) center, we identified a number of lines with potentially useful traits. For example, accession PI 311735, while providing only an average number of seed per plant (4953) produced the greatest yields in overall seed weight at $5.06 \mathrm{~g}$ per plant. This accession also had one of the highest mean oil contents per TSW at 38.2\% and was seen to be faster than average for days to maturity and drying. As results were so encouraging with this line, its use for biotech application was also explored. It was observed that this accession was capable of transformation via traditional floral-dip technology and that glufosinate was an effective agent for seed selection. Thus, observation indicates that accession PI 311735 is a potential line for both breeding and biotech use. Another line of interest was observed through biochemical analysis of the oil, where it was discovered that accession PI 650141 had an erucic acid concentration of $1.83 \%$, which is below the $2 \%$ as required for food consumption. Making this another potentially useful line for breeding efforts. Of interest was the split seen in the camelina population for chromosome number between $n=19$ (24.3\%) and $n=20$ (75.6\%). Our group hypothesized that camelina in its current hexaploidy form may have originated from two divergent but related pathways. In short both events could have begun with an $n=6$ (C. laxa) and $n=7$ (C. hispida) hybridization producing $n=13$ (C. rumelica) like species. Then diverged with the second hybridization of $n=13$ to one or the other original parent such that $n=6+7+6(19)$ or $6+7+7$ (20). From the biotechnological studies, it was discovered that the codA gene worked very efficiently at stunting camelina growth in the presences of 5FC. This should provide a valuable resource for techniques, such as RMCE or CRISPR, where the removal of DNA is a required component for both 
strategies. Finally, even though the sulI selection marker gene only produced a single plant that plant has been shown resistant to sulfa based herbicides (data not shown) and may provide farmers with a way to control weeds while cultivating camelina using conventional treatments. Data is presented in an uncompressed format in both tables for manuscript discussion and as raw data in XLS spreadsheet (Supplemental Tables S1-S4) format. With the variability seen within this collection it is our hope that this information will help direct breeding or biotechnological programs for camelina's future use as biofuel and/or meal sustainable crop.

Supplementary Materials: The following are available online at http://www.mdpi.com/2223-7747/9/5/642/s1, Figure S1. Selectable markers vectors for Camelina; Figure S2. nptII marker assessment for Camelina; Figure S3. hptII marker assessment for Camelina; Figure S4. sulI marker assessment for Camelina; Figure S6. 5-fluorocytosine (5FC) kill curve analysis of Camelina; Figure S7. PCR analysis of putative transgenic PI 311735 and Suneson Camelina; Table S1. Raw Data Oil Analysis; Table S2. Raw Data Compilation Supplemental; Table S3. TSW Stats; Table S4. Total seed weight Stats.

Author Contributions: J.G.T. conceived the idea of the study; S.K.H., M.K., R.C., B.T.H., H.A.Y., C.T., T.M., J.B., and N.J.T. collected data; J.G.T., S.K.H., and M.K. analyzed data and wrote the manuscript. All authors have read and agreed to the published version of the manuscript.

Funding: Research was funded by a grant from the US Department of Agriculture Research BRAG project 2010-33522-21773 and USDA-ARS project 2030-21000-020-00D.

Acknowledgments: Mention of trade names or commercial products in this publication is solely for the purpose of providing specific information and does not imply recommendation or endorsement by the U.S. USDA is an equal opportunity provider and employer. Authors would like to thank the National Genetic Resources Program (NGRP) center collection (https://npgsweb.ars-grin.gov/gringlobal/Search.aspx) for providing the available camelina cultivars and in providing insight for phenotypic evaluation of the species.

Conflicts of Interest: The authors declare that they have no competing interests.

\section{References}

1. Knorzer, K.H. Evolution and spread of gold of pleasure (Camelina sativa S.L.). Ber. Dtsch Bot Ges. 1978, 91, 187-195.

2. Alexander, J.; Glob, P.V. The Bog People: Iron-Age Man Preserved (translated from the Danish by R. Bruce-Mitford). London: Faber and Faber. 198 pp., 76 pls., 2 maps. 50s. Antiquity 1969, 43, 320-321. [CrossRef]

3. Robinson, D.E. Neolithic and Bronze Age Agriculture in Southern Scandinavia-Recent Archaeobotanical Evidence from Denmark. Environ. Archaeol. 2003, 8, 145-165. [CrossRef]

4. Zubr, J. Oil-seed crop: Camelina sativa. Ind. Crop. Prod. 1997, 6, 113-119. [CrossRef]

5. Zubr, J. Unique dietary oil from Camelina sativa seed. Agro Food Industry Hi Tech. 2009, 20, 42-46.

6. Putnam, D.H.; Budin, J.T.; Field, L.A.; Breene, W.M. Camelina: A promising low-input oilseed. In New Crops; Wiley: New York, NY, USA, 1993; pp. 314-322.

7. Vollmann, J.; Moritz, T.; Kargl, C.; Baumgartner, S.; Wagentristl, H. Agronomic evaluation of camelina genotypes selected for seed quality characteristics. Ind. Crop. Prod. 2007, 26, 270-277. [CrossRef]

8. Bansal., S.; Durrett, T.P. Camelina sativa: An ideal platform for the metabolic engineering and field production of industrial lipids. Biochimie 2016, 120, 9-16. [CrossRef]

9. Brown, T.D.; Hori, T.S.; Xue, X.; Ye, C.L.; Anderson, D.M.; Rise, M.R. Functional Genomic Analysis of the Impact of Camelina (Camelina sativa) Meal on Atlantic Salmon (Salmo salar) Distal Intestine Gene Expression and Physiology. Mar. Biotechnol. 2016, 18, 418-435. [CrossRef]

10. Lovett, J.V.; Ryuntyu, M.Y.; Liu, D.L. Allelopathy, chemical communication, and plant defense. J. Chem. Ecol. 1989, 15, 1193-1202. [CrossRef]

11. Betancor, M.B.; Spraque, M.; Sayonova, O.; Usher, S.; Campbell, P.J.; Napier, J.A.; Caballero, M.J.; Tocher, D.R. Evaluation of a high-EPA oil from transgenic Camelina sativa in feeds for Atlantic salmon (Salmo salar L.): Effects on tissue fatty acid composition, histology and gene expression. Aquaculture 2015, 444, 1-12. [CrossRef]

12. Betancor, M.B.; Spraque, M.; Usher, S.; Sayonova, O.; Campbell, P.J.; Napier, J.A.; Tocher, D.R. A nutritionally-enhanced oil from transgenic Camelina sativa effectively replaces fish oil as a source of eicosapentaenoic acid for fish. Sci. Rep. 2015, 5, 8104. [CrossRef] [PubMed] 
13. Betancor, M.B.; Spraque, M.; Montero, D.; Usher, S.; Sayonova, O.; Campbell, P.J.; Napier, J.A.; Caballero, M.J.; Izquierdo, D.R.; Tocher, D.R. Replacement of Marine Fish Oil with de novo Omega-3 Oils from Transgenic Camelina sativa in Feeds for Gilthead Sea Bream (Sparus aurata L.). Lipids 2016, 51, 1171-1191. [CrossRef] [PubMed]

14. Betancor, M.B.; Spraque, M.; Sayonova, O.; Usher, S.; Metochis, C.; Campbell, P.J.; Napier, J.A.; Tocher, D.R. Nutritional Evaluation of an EPA-DHA Oil from Transgenic Camelina sativa in Feeds for Post-Smolt Atlantic Salmon (Salmo salar L.). PLoS ONE 2016, 11, e0159934. [CrossRef] [PubMed]

15. Moloney, A.P.; Woods, V.B.; Crowley, J.G. A Note on the Nutritive Value of Camelina Meal for Beef Cattle. J. Agric. Food Res. 1998, 37, 243-247.

16. Pietras, M.P.; Orczewska-Dudek, S. The effect of dietary Camelina sativa oil on quality of broiler chicken meat. Ann. Anim. Sci. 2013, 13, 869-882. [CrossRef]

17. Peiretti, P.G.; Mussa, P.P.; Prola, L.; Meineri, G. Use of Different Levels of False Flax Camelina sativa L. Seed in Diets for Fattening Rabbits. Livest. Sci. 2007, 107, 192-198. [CrossRef]

18. Habeanu, M.; Hebean, V.; Taranu, I.; Ropota, M.; Lefter, N.A.; Marin, D. Dietary Ecologic Camelina Oil: A Beneficial Source of n-3 PUFA in Muscle Tissue and Health Status in Finishing Pigs. Romanian Biotechnol. Lett. 2011, 16, 5564-5571.

19. Hasan, M.R.; Halwart, M. Fish as Feed Inputs for Aquaculture: Practices, Sustainability and Implications. In FAO Fisheries and Aquaculture Technical Paper; FAO: Rome, Italy, 2009; pp. 1-61, No. 518.

20. Acamovic, T.; Gilbert, C.; Lamb, K.; Walker, K.C. Nutritive value of Camelina sativa meal for poultry. Br. Poult. Sci. 1999, 40, 27. [CrossRef]

21. Colombini, S.; Broderick, G.A.; Galasso, I.; Martinelli, T.; Rapetti, L.; Russo, R.; Reggiani, R. Evaluation of Camelina sativa (L.) Crantz meal as an alternative protein source in ruminant rations. J. Sci. Food Agric. 2014, 94, 736-743. [CrossRef]

22. Russo, R.; Reggiani, R. Glucosinolates and Sinapine in Camelina Meal. Food Nutr. Sci. 2017, 8, $1063-1073$. [CrossRef]

23. Schill, R.S. Camelina Meal Approved for Feedlot Cattle. Biodiesel Magazine, 2009. Available online: http: //biodieselmagazine.com/articles/3928/camelina-meal-approved-for-feedlot-cattle (accessed on 12 May 2020).

24. Karvonen, H.M.; Aro, A.; Tapola, N.S.; Salminen, I.; Uusitupa, M.I.J.; Sarkkinen, E.S. Effect of alpha-linolenic acid-rich Camelina sativa oil on serum fatty acid composition and serum lipids in hypercholesterolemic subjects. Metabolism 2002, 51, 1253-1260. [CrossRef] [PubMed]

25. Schwab, U.S.; Lankin, M.A.; de Mello, V.D.; Manninen, S.M.; Kurl, S.; Pulkki, K.J.; Laaksonen, D.E.; Erkkila, A.T. Camelina Sativa Oil, but not Fatty Fish or Lean Fish, Improves Serum Lipid Profile in Subjects with Impaired Glucose Metabolism-A Randomized Controlled Trial. Mol. Nutr. Food Res. 2018, 62. [CrossRef] [PubMed]

26. Campbell, M.C.; Rossi, A.F.; Erskine, W. Camelina (Camelina sativa (L.) Crantz): Agronomic potential in Mediterranean environments and diversity for biofuel and food uses. Crop. Pasture Sci. 2013, 64, 388-398. [CrossRef]

27. Huber, G.W.; Iborra, S.; Corma, A. Synthesis of transportation fuels from biomass: Chemistry, catalysts, and engineering. Chem. Rev. 2006, 106, 4044-4098. [CrossRef]

28. Buchsenschutz-Nothdurft, A.; Schuster, A.; Friedt, W. Breeding for modified fatty acid composition via experimental mutagenesis in Camelina sativa (L.) Crtz. Ind. Crop. Prod. 1988, 7, 291-295. [CrossRef]

29. Vollmann, J.; Grausgruber, H.; Stift, G.; Dryzhyruk, V.; Lelley, T. Genetic diversity in camelina germplasm as revealed by seed quality characteristics and RAPD polymorphism. Plant. Breed. 2005, 124, 446-453. [CrossRef]

30. French, A.N.; Hunsaker, D.; Thorp, K.; Clarke, T. Evapotranspiration over a camelina crop at Maricopa. Arizona. Ind. Crop. Prod. 2009, 29, 289-300. [CrossRef]

31. Moser, B.R. Camelina (Camelina sativa L.) oil as a biofuels feedstock: Golden opportunity or false hope? Lipid Technol. 2010, 22, 270-273. [CrossRef]

32. Cahoon, E.B.; Shockey, J.M.; Dietrich, C.R.; Gidda, S.K.; Mullen, R.T.; Dyer, J.M. Engineering oilseeds for sustainable production of industrial and nutritional feedstocks: Solving bottlenecks in fatty acid flux. Curr. Opin. Plant. Biol. 2007, 10, 236-244. [CrossRef] 
33. Dalal, J.; Lopez, H.; Vasani, N.B.; Zhaohui, H.; Swify, J.E.; Yalamanchili, R.; Dvora, M.; Lin, X.; Xie, D.; $\mathrm{Qu}, \mathrm{R}$.; et al. Photorespiratory bypass increases plant growth and seed yield in biofuel crop Camelina sativa. Biotechnol. Biofuels 2015, 8, 175. [CrossRef]

34. Haslam, R.P.; Sayanova, O.; Kim, H.J.; Cahoon, E.B.; Napier, J.A. Synthetic redesign of plant lipid metabolism. Plant. J. 2016, 87, 76-86. [CrossRef]

35. Hu, Z.; Wu, Q.; Dalal, J.; Vasani, N.; Lopez, H.O.; Sederoff, H.W.; Qu, R. Accumulation of medium-chain, saturated fatty acyl moieties in seed oils of transgenic Camelina sativa. PLoS ONE 2017, 12, e0172296. [CrossRef]

36. Crowley, J.G.; Fröhlich, A. Factors affecting the composition and use of camelina. Irish Agriculture and Food Development Authority. Teagsac: Dublin, 1998; ISBN 190113866 6. Available online: https: //t-stor.teagasc.ie/handle/11019/1481 (accessed on 12 May 2020).

37. Vollmann, J.; Damboeck, A.; Eckl, A.; Schrems, H.; Ruckenbauer, P. Improvement of Camelina sativa, an underexploited oilseed. In Progress in New Crops; ASHS Press: Alexandria, VA, USA, 1996; pp. 357-362.

38. Guy, S.O.; Wysocki, D.J.; Schillinger, W.F.; Thomas, G.; Chastain, T.G.; Karow, R.S.; Garland-Campbeli, K.; Burke, I.C. Camelina: Adaptation and Performance of Genotypes. Field Crop. Res. 2014, 155, $224-232$. [CrossRef]

39. Ehrensing, D.T.; Guy, S.O. Camelina. In Oregon State Univ. Ext Ser Publ, EM8953-E; Oregon State University: Corvallis, OR, USA, 2008.

40. Obour, A.K.; Sintim, H.Y.; Obeng, E.; Jeliazkov (Zhelijazkov), V.D. Oilseed camelina (Camelina sativa L Crantz): Production systems, prospects and challenges in the USA great plains. Adv. Plants Agric. Res. 2015, 2, 68-76. [CrossRef]

41. Lu, C.; Kang, J. Generation of transgenic plants of a potential oilseed crop Camelina sativa by Agrobacterium-mediated transformation. Plant. Cell Rep. 2008, 27, 273-278. [CrossRef] [PubMed]

42. Liu, X.; Brost, J.; Hutcheon, C.; Guilfoil, R.; Wilson, A.; Leung, S.; Shewmaker, C.; Rooke, S.; Nguyen, T.; Kiser, J.; et al. Transformation of the oilseed crop Camelina sativa by Agrobacterium-mediated floral dip and simple large-scale screening of transformants. In Vitro Cell Dev. Biol. Plant. 2012, 48, 462-468. [CrossRef]

43. Yemets, A.I.; Boychuk, Y.N.; Shysha, E.N.; Rakhmetov, D.B.; Blume, Y.B. Establishment of in vitro culture, plant regeneration, and genetic transformation of Camelina sativa. Cytol. Genet. 2013, 47, 138-144. [CrossRef]

44. Zhang, Y.; Yu, L.; Yung, K.-F.; Leung, D.Y.C.; Sun, F.; Boon, L.L. Over-expression of AtPAP2 in Camelina sativa leads to faster plant growth and higher seed yield. Biotechnol. Biofuels 2012, 5, 19. [CrossRef]

45. Usher, S.; Han, L.; Haslam, R.P.; Michaelson, L.V.; Sturtevant, D.; Aziz, M.; Chapman, K.D.; Sayanova, O.; Napier, J.A. Tailoring seed oil composition in the real world: Optimizing omega-3 long chain polyunsaturated fatty acid accumulation in transgenic Camelina sativa. Sci. Rep. 2017, 7, 6570. [CrossRef]

46. Choudhury, S.R.; Riesselman, A.J.; Pandey, S. Constitutive or seed-specific overexpression of Arabidopsis G-protein c subunit 3 (AGG3) results in increased seed and oil production and improved stress tolerance in Camelina sativa. Plant. Biotechnol. J. 2013, 12, 49-59. [CrossRef]

47. Ju, S.; Go, Y.S.; Choi, H.J.; Park, J.M.; Suh, M.C. DEWAX Transcription Factor Is Involved in Resistance to Botrytis cinerea in Arabidopsis thaliana and Camelina sativa. Frontiers Plant. Sci. 2017, 8, 1210. [CrossRef] [PubMed]

48. Nguyen, H.T.; Silva, J.E.; Podicheti, R.; Macrander, J.; Yang, W.; Nazarenus, T.J.; Nam, J.-W.; Jaworski, J.G.; Lu, C.; Scheffler, B.E.; et al. Camelina seed transcriptome: A tool for meal and oil improvement and translational research. Plant. Biotechnol. J. 2013, 11, 759-769. [CrossRef] [PubMed]

49. Kagale, S.; Koh, C.; Nixon, J.; Bollina, V.; Clarke, W.E.; Tuteja, R.; Spillane, C.; Robinsin, S.J.; Links, M.G.; Clarke, C.; et al. The emerging biofuel crop Camelina sativa retains a highly undifferentiated hexaploidy genome structure. Nat. Commun. 2014, 5, 3706. [CrossRef] [PubMed]

50. Gehringer, A.; Friedt, W.; Luehs, W.; Snowdon, R.J. Genetic mapping of agronomic traits in false flax (Camelina sativa subsp sativa). Genome 2006, 49, 1555-1563. [CrossRef]

51. Kim, C.; Lee, J.H.; Chung, Y.S.; Choi, S.C.; Guo, H.; Lee, T.-H.; Lee, S. Characterization of twenty Camelina spp. accessions by using single nucleotide polymorphism genotyping. Hortic. Env. Biotechnol. 2017, 58, 187-194. [CrossRef]

52. Singh, R.; Bollina, V.; Higgins, E.E.; Clarke, W.E.; Eynck, C.; Sidebottom, C.; Gugel, R.; Snowdon, R.; Parkins, I.A.P. Single-nucleotide polymorphism identification and genotyping in Camelina sativa. Mol. Breed.. 2015, 35, 35. [CrossRef] 
53. Ghamkhar, K.; Croser, J.; Aryamanesh, N.; Campbell, M.; Konkova, N.; Francis, C. Camelina (Camelina sativa L. Crantz) as an alternative oilseed: Molecular and ecogeographic analyses. Genome 2010, 53, 558-567. [CrossRef]

54. Wang, X.; Wu, J.; Liang, J.; Cheng, F.; Wang, X. Brassica database (BRAD) version 2.0: Integrating and mining Brassicaceae species genomic resources. Database (Oxf. ) 2015. [CrossRef]

55. Jiang, W.Z.; Henry, I.M.; Lynagh, P.G.; Comai, L.; Cahoon, E.B.; Weeks, D.P. Significant enhancement of fatty acid composition in seeds of the allohexaploid, Camelina sativa, using CRISPR/Cas9 gene editing. Plant. Biotechnol. J. 2017, 15, 648-657. [CrossRef]

56. Wang, Y.; Yau, Y.-Y.; Perkins-Balding, D.; Thomson, J.G. Recombinase technology: Applications and possibilities. Plant. Cell Rep. 2011, 30, 267-285. [CrossRef]

57. Collier., R.; Thomson, J.G.; Thilmony, R. A versatile and robust Agrobacterium-based gene stacking system generates high quality transgenic plants. Plant. J. 2018, 95, 573-583. [CrossRef] [PubMed]

58. Varshney, R.K.; Nayak, S.N.; May, G.D.; Jackson, S.A. Next-generation sequencing technologies and their implications for crop genetics and breeding. Trends Biotech. 2009, 27, 522-530. [CrossRef]

59. Edwards, D.; Batley, J.; Snowdon, R.J. Accessing complex crop genomes with next-generation sequencing. Theor. Appl. Genet. 2013, 126,1-11. [CrossRef] [PubMed]

60. Huntington, T.; Hasan, M.R. Fish as Feed Inputs for Aquaculture-Practices, Sustainability and Implications: A Global Synthesis. FAO Glossary of Aquaculture . 2009. Available online: http://www.fao.org/3/i1140e/ i1140e1.pdf (accessed on 12 May 2020).

61. Sprague, M.; Betancor, M.B.; Tocher, D.R. Microbial and genetically engineered oils as replacements for fish oil in aquaculture feeds. Biotechnol Lett. 2017, 39, 1599-1609. [CrossRef] [PubMed]

62. Wang, L.; Li, Z.; Qian, W.; Guo, W.; Gao, X.; Huang, L.; Wang, H.; Zhu, H.; Wu, J.-W.; Wang, D.; et al. The Arabidopsis Purple Acid Phosphatase AtPAP10 Is Predominantly Associated with the Root Surface and Plays an Important Role in Plant Tolerance to Phosphate Limitation. Plant. Physiol. 2011, 157, 1283-1299. [CrossRef] [PubMed]

63. Kang, J.; Snapp, A.R.; Lu, C. Identification of three genes encoding microsomal oleate desaturases (FAD2) from the oilseed crop Camelina sativa. Plant. Physiol. Biochem. 2011, 49, 223-229.

64. Bansal, S.; Kim, H.J.; Na, G.; Hamilton, M.E.; Cahoon, E.B.; Lu, C.; Durrett, T.P. Towards the synthetic design of camelina oil enriched in tailored acetyl-triacylglycerols with medium-chain fatty acids. J. Exp. Bot. 2018, 69, 4395-4402. [CrossRef]

65. Walsh, K.D.; Puttick, D.M.; Hills, M.J.; Yang, R.C.; Topinka, K.C.; Hall, L.M. First report of outcrossing rates in camelina [Camelina sativa (L.) Crantz], a potential platform for bioindustrial oils. Can. J. Plant. Sci. 2012, 92, 681685. [CrossRef]

66. Mohammadi, S.A.; Prasanna, B.M. Analysis of genetic diversity in crop plants—Salient statistical tools and considerations. Crop. Sci 2003, 43, 1235-1248. [CrossRef]

67. Martinelli, T.; Galasso, I. Phenological growth stages of Camelina sativa according to the extended BBCH scale. Ann. Appl. Biol. 2011, 158, 87-94. [CrossRef]

68. Waring, M.; Britten, R.J. Nucleotide sequence repetition: A rapidly reassociating fraction of mouse DNA. Science 1966, 154, 791-794. [CrossRef] [PubMed]

69. Thomson, J.G.; Yau, Y.-Y.; Blanvillain, R.; Chiniquy, D.; Thilmony, R.; Ow, D.W. ParA resolvase catalyzes site-specific excision of DNA from the Arabidopsis genome. Transgenic Res. 2009, 18, 237-248. [CrossRef] [PubMed]

70. Thomson, J.G.; Chan, R.; Thilmony, R.; Yau, Y.-Y.; Ow, D.W. PhiC31 recombination system demonstrates heritable germinal transmission of site-specific excision from the Arabidopsis genome. Bmc Biotechnol. 2010, 23, 17. [CrossRef] [PubMed]

71. Thomson, J.G.; Cook, M.; Guttman, M.; Smith, J.; Thilmony, R. Novel sulI binary vectors enable an inexpensive foliar selection method in Arabidopsis. Bmc Res. Notes 2011, 4, 44. [CrossRef]

72. De Oliveira, M.L.; Stover, E.; Thomson, J.G. The codA gene as a negative selection marker in Citrus. SpringerPlus 2015, 4, 264-270. [CrossRef] [PubMed]

73. Shao, M.; Michno, J.M.; Hotton, S.K.; Blechl, A.; Thomson, J.G. A bacterial gene codA encoding cytosine deaminase is an effective conditional negative selectable marker in Glycine max. Plant. Cell Rep. 2015, 34, 1707-1716. [CrossRef]

74. Warwick, S.I.; Al-Shehbaz, I.A. Brassicaceae: Chromosome number index and database on CD-Rom. Pl. Syst. Evol. 2006, 259, 237-248. [CrossRef] 
75. Angelini, L.G.; Moscheni, E.; Colonna, G.; Belloni, P.; Bonari, E. Variation in agronomic characteristics and seed oil composition of new oilseed crops in central Italy. Ind. Crops Prod. 1997, 6, 313-323. [CrossRef]

76. Hutcheon, C.; Ditt, R.F.; Beilstein, M.; Comai, L.; Schroeder, J.; Goldstein, E.; Shewmaker, C.K.; Nguyen, T.; De Rocher, J.; Kiser, J. Polyploid genome of Camelina sativa revealed by isolation of fatty acid synthesis genes. Bmc Plant. Biol. 2010, 10, 233. [CrossRef]

77. Francis, A.; Warwick, S.I. The biology of Canadian weeds. 142. Camelina alyssum (Mill.) Thell.; C. microcarpa Andrz. ex DC.; C. sativa (L.) Crantz. Can. J. Plant. Sci. 2009, 89, 791-810. [CrossRef]

78. Séguin-Swartz, G.; Nettleton, J.; Sauder, C.; Warwick, S.I.; Gugel, R.K. Hybridization potential between the oilseed crucifer Camelina sativa and canola, mustard and related weeds. Plant. Breed. 2013, 132, 390-396. [CrossRef]

79. Julie-Galau, S.; Bellec, Y.; Faure, J.-D.; Tepfer, M. Evaluation of the potential for interspecific hybridization between Camelina sativa and related wild Brassicaceae in anticipation of field trials of GM camelina. Transgenic Res. 2014, 23, 67-74. [CrossRef] [PubMed]

80. Galasso, I.; Manca, A.; Braglia, L.; Martinelli, T.; Morello, L.; Breviario, D. h-TBP: An approach based on intron-length polymorphism for the rapid isolation and characterization of the multiple members of the $\beta$-tubulin gene family in Camelina sativa (L.). Mol. Breed. 2011, 28, 635-645. [CrossRef]

81. Brock, J.R.; Donmez, A.A.; Beilstein, M.A.; Olsen, K.M. Phylogenetics of Camelina Crantz. (Brassicaceae) and insights on the origin of gold-of-pleasure (Camelina sativa). Mol. Phylogenetics Evol. 2018, 127, 834-842. [CrossRef]

82. Sybenga, J.; Verhaar, H.; Botje, D.G.A. Estimating meiotic chromosome pairing and recombination parameters in telocentric trisomics. Genome 2007, 50, 1014-1028. [CrossRef]

83. Kirov, I.; Divashuk, M.; Van Laere, K.; Soloviev, A.; Khrustaleva, L. An Easy 'SteamDrop' Method for High Quality Plant Chromosome Preparation. Mol. Cytogenet. 2014, 7, 21. [CrossRef]

84. Arumuganathan, K.; Earle, E.D. Nuclear DNA content of some important plant species. Plant. Mol Biol. Rep. 1991, 9, 208-218. [CrossRef]

(C) 2020 by the authors. Licensee MDPI, Basel, Switzerland. This article is an open access article distributed under the terms and conditions of the Creative Commons Attribution (CC BY) license (http://creativecommons.org/licenses/by/4.0/). 